\title{
Antiphon : la nature avec la loi et sans l' intérêt personnel
}

\section{Catherine Darbo-Peschanski}

\section{(2) OpenEdition}

1 Journals

Édition électronique

URL : https://journals.openedition.org/philosant/906

DOI : 10.4000/philosant.906

ISSN : 2648-2789

Éditeur

Éditions Vrin

\section{Édition imprimée}

Date de publication : 1 novembre 2013

Pagination : 173-223

ISBN : 978-2-7574-0591-8

ISSN : 1634-4561

\section{Référence électronique}

Catherine Darbo-Peschanski, «Antiphon : la nature avec la loi et sans l' intérêt personnel », Philosophie antique [En ligne], 13 | 2013, mis en ligne le 01 novembre 2018, consulté le 02 décembre 2022. URL : http://journals.openedition.org/philosant/906 ; DOI : https://doi.org/10.4000/philosant.906

\section{(c) $(1) \&$}

Creative Commons - Attribution - Pas d'Utilisation Commerciale - Pas de Modification 4.0 International - CC BY-NC-ND 4.0

https://creativecommons.org/licenses/by-nc-nd/4.0/ 


\section{ANTIPHON : \\ LA NATURE $A V E C$ LA LOI ET SANS L'INTÉRÊT \\ PERSONNEL}

Catherine DARBO-PESCHANSKI

Laboratoire d'Anthropologie Sociale, CNRS, Paris

RÉSUMÉ. L'article adopte un point de vue unitaire selon lequel sont interrogés à la fois les témoignages sur la physique d'Antiphon, les fragments du Peri aletheias, ainsi que les plaidoyers et, parmi les œuvres qu'on a longtemps considérées comme «morales », le Peri homonoias. Une analyse détaillée d'Aristote, Physique II, 193a10-27, et de ses commentateurs anciens, ainsi que du Peri Aletheias (F44a Pendrick, col. II, 23-30) sert de point de départ à une mise à l'épreuve ultérieure de la thèse par la lecture suivie de l'ensemble. Il s'agit de soutenir qu'Antiphon affirme et montre la superposition nécessaire du nomos sur la physis en établissant leur inégal statut ontologique, de substrat matériel d'une part, de forme accidentelle surajoutée d'autre part. Cette interprétation trouve son prolongement dans l'analyse des deux xympheronta (celui de la nature et celui de la norme), et de l'heautos qui leur est couramment associé comme le «soi-même» le serait à «l'utile», selon l'idée qu'il serait question de combattre la norme au nom de l'intérêt personnel supposé, quant à lui, naturel. Le xympheron est plutôt pris ici comme « ce qui constitue », en anthropos, si c'est selon la nature ; en membre d'une communauté politique, si c'est selon la norme. La dépendance mutuelle de la physis et du nomos, comme de leurs xympheronta respectifs, fait alors que le naturel ne se révèle qu’à l'occasion de circonstances accidentelles et éphémères qui, un moment, isolent un membre d'un groupe politique de ce groupe-là, ou au prix d'une opération intellectuelle qui, pour un temps également, fait abstraction de l'œuvre constituante du nomos.

SUMMARY. The paper examines from a unitary point of view the testimonies on Antiphon's physics, the fragments of the Peri aletheias, the forensic speeches, and the Peri homonoias that has long been regarded as a moral treatise. A detailed analysis of Aristotle's Physics II. 193a10-27, of the ancient commentaries on this passage and of Peri Aletheias (F44a Pendrick, col. II, 23-30) is used to put to the test, throughout the paper, the thesis that Antiphon holds the necessary overlaying of the nomos on the nature, and their unequal ontological status of material substratum, on the one hand, and accidental shape, on the other. The discussion moreover continues about the meaning of the two xympheronta (one based on physis, the other on nomos) and of the heautos, usually associated with them as "the useful" would be associated with "the selfhood", in the belief that nature would fight nomos on behalf of self-interest. But the xympheron may be interpreted as "constitutive", either "of" the anthropos as regards physis or "of" the member of a political community, as regards nomos. Because 
of the mutual dependance of physis and nomos, and of their xympheronta as well, nature only appears in purely accidental and ephemeral circumstances, when, for a limited time, a member of a political group is isolated from it, or by means of an intellectual operation consisting in disregarding, also for a limited time, the constitutive action of nomos. 
Nos sources grecques mentionnent le nom d'Antiphon, soit sans autre précision, soit en lui adjoignant, ici le qualificatif de sophiste, là de logographe, ailleurs de poète, de logothérapeute, ou encore d'interprète des rêves. De là certains, qu'on appelle parfois « séparatistes », infèrent l'existence de plusieurs Antiphon dans le dernier tiers du $V^{\mathrm{e}}$ siècle avant notre ère. Le problème, pour ces interprètes, est alors de savoir en quel nombre ils sont $^{1}$. Généralement ils en retiennent deux, voire trois : le sophiste, le poète et l'orateur du dème de Rhamnonte ${ }^{2}$. D'autres, en revanche, les « uni-

1. La question de l'identité d'Antiphon a souvent été traitée de façon très soigneuse. Parmi les exposés relativement récents les plus documentés, on citera l'article de Narcy 1989. Après examen des sources anciennes, l'auteur constate (p. 230) qu'on a affaire à deux types de données : d'une part, il y a bien deux Antiphon, morts en des lieux et à des dates différents, identifiables l'un par son activité oratoire, notamment aux côtés des Quatre Cents, l'autre par son œuvre poétique; d'autre part, sous le nom d'Antiphon, se rangeaient des œuvres de styles suffisamment différents, aux yeux des Anciens, pour faire penser à deux auteurs eux-mêmes différents. Le reste est affaire d'interprétation des modernes qui, à coup de recoupements de sources et d'assimilation plus ou moins fondées, ont construit la distinction entre «Antiphon l'orateur » et « Antiphon le sophiste ». D'où la création d'un nouveau front séparant les « unitaristes » et les « séparatistes » (et, secondairement, à partir de E. Bignone, qui écrit sur le thème de 1919 à 1923, les tenants d'un Antiphon oligarque et d'un Antiphon démocrate). Pour ceux qui, dans les quarante dernières années, se placent dans la perspective unitariste, mentionnons, par exemple, après Morrison 1972, Cassin 1995, p. 151-191, Hourcade 2001 ou Gagarin 2002. Desclos 2009, p. 163-172, opte aussi pour une lecture de tendance unitariste. Le point de vue séparatiste a été soutenu récemment par Pendrick 2002. Toutefois Decleva Caizzi 1985 signale que, depuis la Seconde Guerre Mondiale, la tendance interprétative s'est inversée au profit de la lecture unitariste qu'elle-même choisit en mettant constamment les traités Sur la vérité et Sur la concorde en rapport avec les Tétralogies. Elle salue, par ailleurs, le recueil de R. Kent Sprague 1972 comme donnant, grâce à Morrison (p. 106-240), la première traduction anglaise de l'ensemble du matériel antiphonien.

2. Parmi les témoignages anciens les plus troublants pour qui voudrait distinguer les deux Antiphon, figure celui d'Hermogène de Tarse (Пвpì I $\delta \dot{\varepsilon} \omega \nu$ B, p. 399, 18 - 401, 23 Rabe) qui, à propos des formes de style, cite le témoignage antérieur du grammairien Didyme. Celui-ci, dit Hermogène, faisait état de plusieurs Antiphon mais, parmi ceux qui ont pratiqué la sophistique, mentionnait aussi bien l'orateur, auteur des discours sur les cas de 
taristes », veulent bien admettre que le poète ne peut, pour des raisons chronologiques $^{3}$, se confondre avec le rhéteur mais soutiennent que le sophiste et l'orateur ne font qu'un ${ }^{4}$. Notre position sera un peu différente puisque le point de vue unitariste sera d'abord pour nous un choix de méthode. En prenant le parti de parler d'Antiphon au singulier et en considérant comme un ensemble les textes réunis sous ce nom, il s'agira seulement, en effet, de ne pas s'arrêter trop tôt aux oppositions consacrées par une longue tradition de commentaires. On citera, par exemple, celle qui séparerait deux positions politiques : l'une démocratique et l'autre oligarchique; ou celle qu'on pourrait voir entre deux styles : l'un âpre et abrupt, l'autre plus lié et plus souple, mais surtout, pour ce qui nous préoccupe, entre la nature et la loi $^{5}$ et subsidiairement l'individu et le groupe. La position unitariste sera donc adoptée, en quelque sorte, comme un moyen de tendre au plus loin l'effort d'analyse.

Notre lecture tentera prioritairement une interprétation non polémique des rapports entre la nature et la loi chez Antiphon ${ }^{6}$, mais aussi s'attachera à

meurtres, de discours d'assemblée et autres discours de ce type, que le devin et interprète des rêves, considéré aussi comme l'auteur du Peri aletheias, du Peri homonoias, du Politikos et de discours d'assemblée. On peut ajouter ce facteur de trouble supplémentaire que, si l'on n'admet pas de confusion entre les listes d'œuvres qu'offrent les manuscrits, les discours d'assemblée, voire le Politikos, sont attribués à l'un comme à l'autre. Quant aux critères stylistiques, Hermogène lui-même renonce à soutenir qu'il y ait deux Antiphon en s'appuyant sur eux car, si l'orateur a bien été le maitre (ou l'élève) de Thucydide, le style de l'auteur de la Guerre du Péloponnèse se rapproche beaucoup plus de celui de l'auteur du traité Sur la vérité que de celui de l'orateur.

3. L'orateur/logographe du dème de Rhamnonte a été condamné à mort et exécuté en 411, à l'issue du procès intenté contre lui pour sa participation au coup d'État des Quatre Cents, tandis que, pour beaucoup d'auteurs anciens, tels Aristote (Rhét. II, 6, 1385a10-13), Plutarque (De Stoic. Repugn. 1051 C-D), le Pseudo-Plutarque (Vitae Or. X, 833 B-C) et Philostrate (Vit. Soph. 15-29, Kayser4), le poète se trouvait alors chez le tyran de Syracuse, Denys, par lequel il aurait été finalement mis à mort.

4. L'un des points de vue les plus intéressants et originaux en la matière est celui qu'adopte Cassin 1995, p. 151-191. L'auteur parvient à la conclusion qu'il n'y a pas un Antiphon rhéteur et un Antiphon sophiste, mais un seul Antiphon, en analysant les présupposés de l'analyse séparatiste, en deçà des arguments explicitement avancés. Ils tiennent, selon elle, aux évaluations contradictoires dont la sophistique elle-même fait l'objet, entre philosophie et rhétorique de mauvais aloi, et auxquelles fait écho le nom même d'Antiphon : « celui qui parle contre », c'est-à-dire « qui peut retourner les arguments dans un procès ou dans une controverse ontologique ».

5. Pour soutenir le contraire, on allègue généralement deux phrases du Peri aletheias:

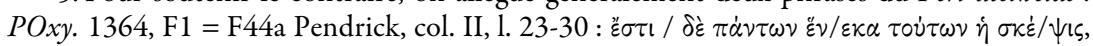

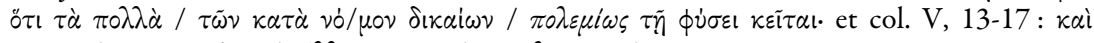

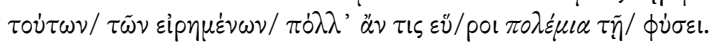

6. Gagarin 2002, n. 1, p. 69 a également choisi cette voie en arguant qu'il n'y a pas de règles de la nature pour s'opposer au nomos, tout simplement parce que la nature ne promeut aucune règle et que l'hostilité peut s'entendre seulement au sens où le nomos, « en im- 
cerner dans ses textes la notion de xympheron. Celle-ci, qui fait son apparition dans le grec du dernier tiers du $\mathrm{V}^{\mathrm{e}}$ siècle avant notre ère et dont usent abondamment Thucydide ou, de manières qui demandent à être distinguées de celle d'Antiphon, le Thrasymaque et, dans une moindre mesure, le Calliclès de Platon, semble en effet utile pour dessiner la place propre d'Antiphon dans la pensée de son temps. De plus, intervenant au cœur de la réflexion sur les rapports entre la nature et la loi, elle ne manque pas d'être utilisée, pour étayer la soi-disant opposition entre « l'individu » ou « le soi-même » (self) et le groupe, sans éclaircissements suffisants de son sens dans le cadre antiphonien ${ }^{7}$ ni examen de ses implications dans celui, plus large, d'une étude anthropologique et historique du « soi-même ».

\section{Nomos-Pbysis : une alliance dissymétrique mais nécessaire}

À n'en pas douter, dans le Peri aletheias, Antiphon procède à une confrontation aussi minutieuse que systématique du nomos et de la physis. Mais, dans une telle confrontation, il reste à éviter de prendre un travail de différenciation conceptuelle visant à élaborer progressivement et systématiquement les deux notions, ainsi qu'à penser leurs rapports asymétriques, pour un exposé dogmatique qui se contenterait d'asserter leur irréductible antagonisme et d'énumérer les diverses formes de celui-ci.

C'est toute la question du statut du texte du Peri aletheias qui se pose alors et notamment celui des trois fragments de papyrus qui en ont été le plus récemment découverts ${ }^{8}$. Un passage de ceux-ci, en particulier, constitue en quelque sorte la croisée des deux chemins d'interprétation entre lesquels il faut choisir. Il s'agit de deux phrases du POxy. 1364, F1 = F44a Pendrick, col. II, 1. 23-30 :

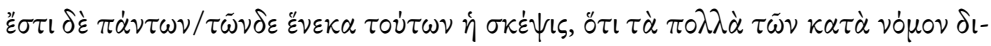
$\kappa \alpha i \omega \nu \pi 0 \lambda \varepsilon \mu i \omega \varsigma \tau \tilde{\eta} \phi \dot{\sigma} \sigma \varepsilon l ~ \kappa \varepsilon \tilde{i} \tau \alpha l$.

posant des exigences supplémentaires (epitheta) à la nature », vient restreindre la liberté d'action qu'elle donne mais sans forcément la contredire ou demander qu'on la viole. Nous reviendrons ultérieurement sur ces différents points.

7. C'est le reproche qu'on peut adresser à l'ouvrage de Nill 1985 et plus encore à celui de Farenga 2006. En revanche, y échappe partiellement celui de Hourcade 2001 qui, s'agissant d'Antiphon, anticipe les thèses exposées dans Ead. 2009, chap. IX, « Antiphon démocritéen $\gg$, p. 202-266. L'auteur veut en effet démontrer que l'individu antiphonien doit s'entendre comme l'atome dans le système démocritéen. Nous aurons cependant à discuter cette position.

8. Jusqu'au début du $\mathrm{XX}^{\mathrm{e}}$ siècle, on n'avait du traité que des fragments tirés d'exposés doxographiques ou des citations de lexicographes comme Harpocration, mais la connaissance d'Antiphon a été considérablement accrue depuis lors par la découverte de plusieurs fragments de papyrus : POxy. 1364 et 1797 , publiés en 1915 et 1922 par B. Grenfell et A. Hunt ; POxy. 3647, publié par M. S. Funghi en 1984. 
J. S. Morrison $(1972)^{9}$ : This is exactly what the present line of argument is about, to demonstrate that many of the things which are just according to the law are at variance with nature.

G. Bastianini, F. Decleva Caizzi $(1989)^{10}$ : L'indagine su queste cose avviene per questo, perché la maggior parte di ciò che è giusto secondo la legge si trova ad essere ostile alla natura.

M. Ostwald $(1990)^{11}$ : The reason for our inquiry into these matters is that the majority of things sanctioned as just in terms of the law are inactments inimical to nature.

M. Gagarin (2002) ${ }^{12}$ : My inquiry into these things is prompted by the fact that most things that are just according to law are hostile to nature.

G. J. Pendrick $(2002)^{13}$ : The examination is being conducted for this reason : because the majority of what is just according to law and convention is hostile to nature.

M.-L. Desclos $(2009)^{14}$ : L'examen de ce qui précède révèle que c'est principalement pour ces raisons : à savoir que la majorité des dispositions justes selon la loi se trouve en lutte avec la nature.

Comme on le voit, les traducteurs, pour ne citer que les plus récents, sont, d'une manière ou d'une autre, d'avis qu'Antiphon asserte ici l'hostilité qui oppose effectivement le juste selon la loi à la nature, sans rien voir qui vienne contredire cette assertion, ou même la nuancer, ni qu'il n'est pas question ici de «toutes » les dispositions normatives ${ }^{15}$ ni qu'une formule conditionnelle est utilisée dans les lignes 13-17 de la colonne $\mathrm{V}$, qui font écho à POxy. 1364, F1 = F44a Pendrick, col. II, 1. 23-30 :

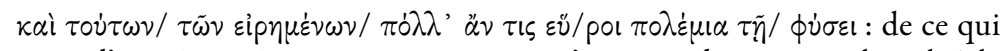
vient d'être évoqué on pourrait trouver qu'une grande partie est hostile à la nature.

Pourtant une autre lecture peut être proposée selon laquelle l'hostilité n'est pas posée comme un fait mais constitue plutôt l'objet problématique d'une étude critique à mener dans la suite du texte. Pour le dire vite, nous pensons que les notions de nomos et de physis sont élaborées en regard précisément parce que la réalité du monde (son aletheia) résulte de leur nécessaire, quoique complexe et inégale, alliance, selon des modalités que le traité s'emploie à analyser pas à pas.

9. Morrison 1972, p. 219.

10. Bastianini \& Decleva Caizzi 1989, p. 202.

11. Ostwald 1990, p. 294.

12. Gagarin 2002, p. 67.

13. Pendrick 2002, p. 163

14. Desclos 2009, p. 199.

15. Comme Bastianini \& Decleva Caizzi 1989 le notent, p. 206, après Moulton 1972, notamment p. 333, et Dillon 1984, notamment p. 129. 


\section{1 La skepsis d'Antiphon}

Pour mesurer cela, il convient de s'interroger conjointement sur le sens à donner au mot skepsis dans POxy. 1364, F1 = F44a Pendrick, col. II, 1. 23-30 et sur la syntaxe de cette phrase.

Le verbe skeptomai apparaît très tôt dans la langue grecque puisqu'on le rencontre dès les poèmes homériques mais, en l'état actuel de notre documentation, il faut attendre les traités hippocratiques et Platon pour voir le nom skepsis s'imposer avec quelque fréquence.

Pour l'ensemble du champ lexical, on ajoutera toutefois avec Lambros Couloubaritsis ${ }^{16}$ que, malgré sa présence précoce, il dénote « un impensé » de la philosophie grecque, avant que n'advienne sa théorisation sceptique. La question que se pose l'auteur est en effet de savoir pourquoi, alors que, dans ce domaine, la pensée critique s'affirme très fortement dès Parménide, c'est-à-dire bien avant la sophistique et la philosophie socratique puis platonicienne, la conceptualisation du skeptesthai n'a pas l'ampleur de celle dont fait l'objet cette autre forme de pensée qu'est le noein. En réponse, il avance l'hypothèse que la conception grecque de la réflexion a pris deux directions séparées: d'une part, la recherche pratique qu'Aristote a problématisée comme «délibération » (bouleusis) dans le cadre de sa théorie de l'acte; d'autre part, la définition sceptique du skeptesthai. En dépit de la forte présence du vocabulaire de la skepsis et du skeptesthai chez Platon, le retard de sa problématisation serait imputable, selon L. Couloubaritsis, à la préexistence d'une forte pratique institutionnalisée de la délibération civique.

Pour notre propos, il importe seulement de noter qu'Antiphon utilise la notion de skepsis à un moment où elle ne renvoie pas encore, autrement que sous la forme d'une lointaine préfiguration (si d'aventure un tel langage a un sens), à sa stricte acception sceptique, autrement dit, à la confrontation des phénomènes et des noumènes qui fasse apparaître la force égale des objets perçus et des raisonnements, et impose par là la suspension de l'assentiment génératrice de tranquillité $e^{17}$; pas plus que, comme nous le verrons ultérieurement, les comparatifs de supériorité ou d'infériorité niés qu'Antiphon multiplie dans le Peri aletheias ne sont assimilables à l'instrument sceptique de mise en équilibre préparant la suspension de l'assentiment que

16. Couloubaritsis 1990.

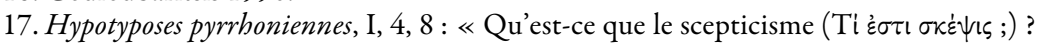

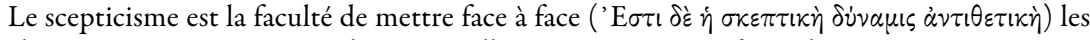
choses qui apparaissent aussi bien que celles qui sont pensées, de quelque manière que ce soit

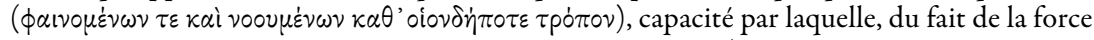

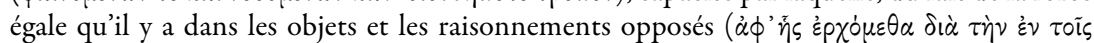

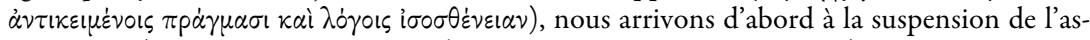

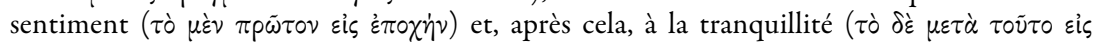
$\alpha \tau \tau \alpha \rho \xi i \alpha \nu) . ~ \gg$ (Traduction Pellegrin 1997) 
sont les formules : «pas plus que» (ou mallon), «en rien plus que» (ouden mallon).

Soit donc le skeptesthai et la skepsis d'avant les sceptiques. On remarquera que, dans l'épopée homérique, skeptomai renvoie exclusivement à la saisie d'une information visuelle ou, plus largement, sensible. Ainsi Hector est présenté ${ }^{18}$ tout à l'écoute (skeptet') du sifflement des flèches et du bruit sourd des armes et Ajax, de son côté, invite Ménélas à observer pour voir :

Regarde (skepteo), Ménélas, nourrisson de Zeus, si tu n'aperçois pas (ai ke ideai) encore vivant Antiloque, le fils magnanime de Nestor ${ }^{19}$. (Il. XVII, 652.)

Quant à l'Odyssée, elle emploie uniquement l'ajectif verbal periskeptos, dans l'expression formulaire periskeptoi en choroi : « en un endroit bien protégé (d'être observable de tous côtés) », qu'elle se rapporte à la chambre de Télémaque $(1,426)$, à la maison de Circé $(10,211$ et 253) ou à la cour de la maison d'Eumée $(14,6)$. Mais le Corpus Hippocratique, dans un de ses traités les plus anciens et susceptible d'être sensiblement contemporain de ceux d'Antiphon, l'Ancienne Médecine, témoigne que, dès la fin du V $\mathrm{V}^{\mathrm{e}}$ siècle avant notre ère, le verbe skeptomai prend les deux valeurs d'examen empirique et d'examen théorique. Il y a, dit en effet $A M 4,2$ et 5, 1, d'un côté, une médecine courante, spontanée, et, de l'autre, un art à part entière. Communément, en effet, remarque le médecin, dans un domaine où personne n'est totalement profane, mais où chacun en sait un peu, par nécessité ou par usage, personne ne mérite le titre de spécialiste de l'art (technites) :

Après que ce fut une découverte importante et le fruit d'une observation (skepsis) et d'une pratique (techne) démultipliée, ce qu'il y a de sûr, c'est que, encore de nos jours, ceux qui s'occupent de l'exercice et de l'entraînement des athlètes ajoutent sans cesse quelque découverte en appliquant la même méthode dans leur recherche visant à déterminer quels sont les aliments et les boissons requis.

Mais à la skepsis, observation empirique, il juxtapose un emploi de skeptesthai pour désigner l'opération théorique par laquelle, en l'occurrence, repérer les différences des deux médecines et définir celle qui est un art, porté par des spécialistes et prenant en compte tous les malades au lieu de s'en tenir au seul régime des athlètes :

Mais examinons aussi (skepsesthai) la médecine reconnue comme telle, celle qui a été découverte autour des malades.

18. Il. XVI, 361

19. Traduction Mazon 1938. 
On serait donc fondé à penser que, chez Antiphon, dans le dernier tiers $\mathrm{du} \mathrm{V}^{\mathrm{e}}$ siècle avant notre ère, il est possible de rencontrer le vocabulaire de la skepsis avec le sens d'examen théorique.

Platon vient, plus tard, confirmer le lien entre le sens d'examen sensible de ce vocabulaire (élargi à celui, de même racine, du skopein) dans le domaine médical et son sens d'examen théorique, tout en montrant ce qu'il peut y avoir de métaphorique dans le rapport des deux. Nous sommes dans le Protagoras, 352a2-b2. Socrate veut étudier ce qu'il en est de l'agréable et du bien. Il fait alors une comparaison :

Comme si un homme, examinant (skopon) à partir de l'apparence extérieure soit la santé de quelqu'un soit son aptitude aux exercices physiques, en voyant son visage ou le bout de ses mains, disait : « Découvre-moi ta poitrine et ton dos afin que je puisse mieux t'examiner (episkepsomai). Eh bien, c'est justement quelque chose d'analogue que je réclame en vue de mon examen (pros ten skepsin). [...] Découvre-moi quelle est ta disposition au regard du savoir.

C'est encore chez Platon qu'on trouve de quoi comprendre plus précisément ce qu'il en est de l'examen théorique. Comme le montre L. Couloubaritsis, on peut conclure qu'avant la théorisation sceptique, il revient à la skepsis et au skeptesthai de renvoyer, d'une façon assez souple, voire lâche, à un acte de pensée par lequel on étudie un objet de deux ou plusieurs points de vue possibles. Il ne s'agit donc pas forcément d'établir un régime d'opposition binaire mais tout autant de déployer un questionnement complexe dans plusieurs directions. C'est ainsi que se nomme skepsis le thème général d'une recherche dialectique qui, pour aboutir (et parfois échouer) devra se diviser et se ramifier en maintes enquêtes subordonnées. Dans les $\operatorname{Lois}^{20}$, par exemple, après la digression initiale sur l'ivresse et la musique qui a permis de franchir un première étape, Mégillos rappelle qu'il s'agit de la législation (peri tes nomothesias skepsis) et se dit prêt à faire une « une longue route » pour mener cette recherche, laquelle effectivement se déploiera d'examen d'étape en examen d'étape durant quelque dix livres. Dans la République, la manière dont la justice et l'injustice prennent naissance dans une cité ${ }^{21}$ est déclarée « objet de toutes les recherches » du dialogue et Socrate, en proposant d'y revenir quand il aborde pour commencer la question de l'éducation des gardiens, demande de ne pas renoncer à cette skepsis, « quelque longue qu'elle puisse être ». En Sophiste 226b7-c11, alors que l'Étranger demande de but en blanc à Théétète d'examiner (skopein) s'ils peuvent tous deux distinguer deux formes dans l'art de trier, Théétète se plaint de ne 
pouvoir répondre immédiatement car cet examen qui ne se subdivise pas en recherches intermédiaires lui paraît «trop rapide ». L'Étranger consent donc à l'interroger pas à pas sur des divisions qui le mèneront à répondre.

De manière analogue, et avec tout autant de souplesse, il semble qu'on puisse interpréter la skepsis d'Antiphon comme l'examen théorique multiforme et ramifié de la question de savoir si la plupart des dispositions de la loi entretiennent avec la nature un rapport d'hostilité.

Il reste alors à se demander si la syntaxe de la phrase du Peri aletheias où est évoquée la skepsis autorise une telle interprétation et, une fois encore, parmi les textes antérieurs à la théorisation sceptique, c'est dans l'œuvre de Platon qu'on trouve le plus matière à mener l'enquête, en raison de la relativement grande fréquence avec laquelle le mot y est employé. L'examen de tel ou tel objet peut se dire skepsis ou episkepsis + peri commandant un nom au génitif ou + peri et un nom à l'accusatif ou + une propossition interrogative indirecte ou $+e p i$ et un nom à l'accusatif ou seulement + un nom au génitif ou + un génitif commandé par heneka: « en vue de ».

De la dernière expression, les Lois fournissent en 894a-b un exemple clair. L'Athénien vient de demander : « N'avons-nous pas, amis, énoncé là et dénombré toutes les espèces du mouvement sauf deux ? 》 « Lesquelles donc ? », interroge Clinias. À quoi l'Athénien répond par la formule suivante : «Tout juste celles, mon bon, auxquelles sera consacré tout l'examen (pronom au génitif + heneka +skepsis) qui est le nôtre maintenant. » « Parle clair », rétorque Clinias. Et l'Athénien de répondre en reprenant la construction nom au génitif + heneka : « N'était-ce pas à l'âme ? 》

La phrase d'Antiphon, quant à elle, adopte, semble-t-il et cette construction et celle, plus simple, de skepsis + génitif. Dans le POxy 1787, on lit

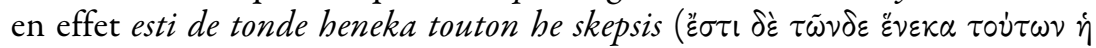
$\sigma \kappa \varepsilon^{\prime} \psi(\zeta)$, lecture adoptée par Diels ${ }^{22}$ seul, puis par Diels et Kranz dans les $F V S$. Cependant, ligne 24 , on trouve aussi un pan $(\pi \tilde{\alpha} \nu)$ subadscrit qui a conduit Diels à ajouter devant tonde $(\tau \tilde{\omega} \nu \delta \varepsilon)$ un pan $\langle\operatorname{tos}\rangle(\pi \dot{\alpha} \nu<\tau \omega \varsigma\rangle)$, ce qu'accepte Untersteiner ${ }^{23}$ mais que refusent Bastianini et Decleva Caizzi ${ }^{24}$. Quant à Pendrick ${ }^{25}$, il choisit de fondre le pan adscrit et le tonde en un panton, ce qui donne : esti de panton heneka touton he skepsis ( $\tilde{\varepsilon} \sigma \tau \iota \delta \dot{\varepsilon} \pi \dot{\alpha} \nu \tau \omega \nu$

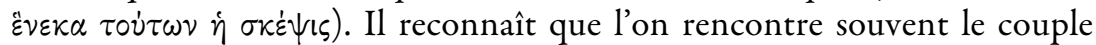

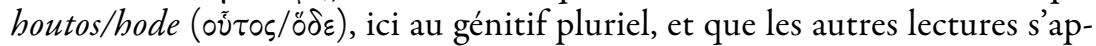
puient sur cette association mais il ajoute aussitôt que le syntagme tonde

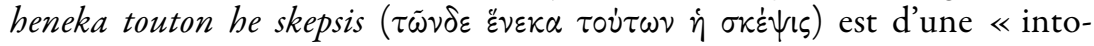

22. Diels 1916, p. 933.

23. Untersteiner 1962, p. 79-80.

24. Bastianini \& Decleva Caizzi 1989, p. 206. Les auteurs préfèrent la lecture originaire du papyrus avant correction, même si c'est la solutio difficilior.

25. Pendrick 2002, p. 327. 


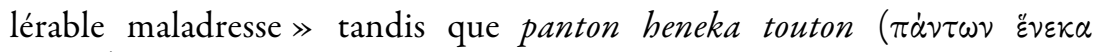
$\tau o \dot{\tau} \omega \nu)$ est bien attesté par ailleurs. Et de citer à l'appui de cela Platon, République, 417b et 526c, Xénophon, Cyropédie, III, 3, 27 ou encore Jamblique 4,4 . Kerferd ${ }^{26}$ avait accepté une leçon semblable à celle de Pendrick, en voyant là une manière de renvoyer à la discussion précédente. Mais Pendrick reste préoccupé par le rapport à établir entre panton heneka touton

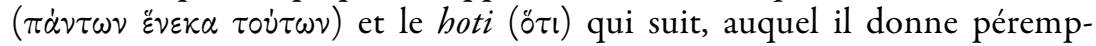
toirement le sens causal de «parce que ». Il se résout finalement à penser

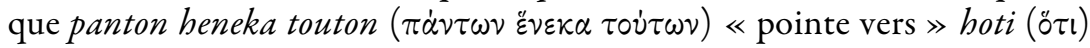
d'une manière analogue à celle qu'on trouve dans le plaidoyer d'Antiphon Sur le meurtre d'Hérode (V, 88, 6). Reportons-nous donc à ce passage. L'orateur vient d'affirmer que, dans une cause de meurtre, une sentence, même injuste, fait loi et prévaut sur la justice et la vérité, si bien que personne n'oserait aller contre le jugement rendu : ni celui qui a été condamné à tort, ni celui qui est acquitté à tort. Et de poursuivre :

C'est pour cela que/avec cela en vue que (pronom au génitif + heneka: $\alpha \dot{\tau} \tau \tilde{\omega} \nu \delta \dot{~} \tau \circ \dot{\tau} \tau \tilde{\omega} \nu \tilde{\varepsilon} \nu \varepsilon \kappa \alpha)$ les lois, les serments, les parties de victimes, les interdictions et, en général, tout ce qui se produit pour juger les actions (nom au génitif + heneka: $\tau \tilde{\omega} \nu \delta i \kappa \omega \nu \tilde{\varepsilon} v \varepsilon \kappa \alpha$ ) pour meurtre est bien différent de ce qui se produit pour les autres causes, parce que (hoti: ö $\tau$ ) les faits où il y a grand risque requièrent plus que les autres une sentence juste.

Peut-on ne pas tenir compte de la nuance finale que prend le syntagme génitif + heneka $^{27}$ et affirmer que, dans ce texte, il pointe vers hoti plutôt que vers ce qui vient d'être énoncé ? Si c'est bien à ce qui précède qu'il fait référence, il s'agirait en effet de dire que c'est pour tenir compte du fait que la justice et la vérité peuvent céder devant la force de loi qu'a la sentence dans des affaires de meurtres, même injustement jugées, que, pour les juger (autre occurrence de génitif theneka à nuance finale qui, en ce cas, ne viendrait pas se distinguer de la première), on a recours à des procédures particulières et différentes de celles qui valent pour les autres causes. Ce serait donc la différence des procédures qui serait expliquée dans un premier temps, tandis que la proposition causale introduite par hot $i$ viendrait, quant à elle, dans un second temps, apporter une explication mais, cette fois, plutôt à la nécessité d'une sentence juste pour les cas de meurtre: cette explication serait l'importance du danger encouru alors par les parties. Certes, ce sont là des nuances peut-être trop subtiles au sein de ce qui constitue, somme

26. Kerferd 1956-1957, p. 28-29.

27. Humbert 1972, p. 330, atteste que heneka a le sens de « à cause de »lié à celui de « en vue de ». Il possède en effet comme hekati, « par la volonté de », un élément nominal exprimant la volonté et se rattachant à hekon, «de gré». Cette «préposition improprement dite » reposerait sur hen (une chose) et heka(t) (volonté). 
toute, un même mouvement argumentatif de nature causale et Pendrick ne

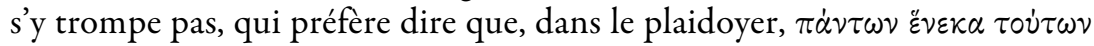
« pointe vers $»^{28} 0 \tilde{\tau} \mathrm{t}$, autrement dit parler d'un lien sémantique, plutôt que d'une corrélation syntaxique avérée. Mais en ce cas, il ne peut se servir de ce passage du plaidoyer pour se prononcer sur la syntaxe de celui du Peri aletheias au sujet duquel, au demeurant, il se montre très circonspect, en constatant que le lien entre panton heneka touton et hoti de sens causal y est obscur $^{29}$.

Quoi qu'il en soit, l'énoncé d'Antiphon dans le Peri aletheias présente la particularité, que n'a pas le texte du plaidoyer, d'employer le mot skepsis à côté du syntagme génitif + heneka dans une formule bien attestée par ailleurs pour désigner le thème envisagé dans la recherche ou la réflexion. De plus, en grec, hoti n'a pas nécessairement une valeur causale mais peut simplement venir développer le contenu d'une expression avec le sens de « autrement dit », « à savoir que » et, pour le texte qui nous occupe, il développerait le touton qui précède skepsis. On obtiendrait donc la traduction suivante :

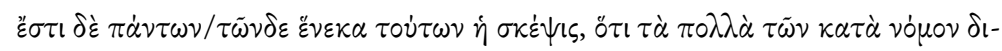

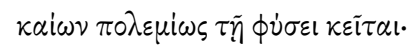

Sur ces questions/en vue de traiter ces questions (qui nous ont préalablement occupés), voici ce qu'il y a lieu de soumettre à l'examen : que la plupart des dispositions justes selon la loi sont en position d'hostilité vis-àvis de la nature.

Antiphon annoncerait donc ici un programme de recherche au terme duquel l'hostilité de la plupart des dispositions du juste selon la loi et de la nature sera questionnée plus qu’affirmée sans détour. En ce cas, il ne se rangerait pas aux côtés de ceux qui, comme Calliclès chez Platon, soutiennent, quant à eux pour de bon, que «le plus souvent, ce sont choses contraires que la nature et la loi $\gg^{30}$, mais demanderait plutôt à voir ce qu'il en est.

\section{2 La Physis selon Antiphon}

Depuis les récentes découvertes des Papyri d'Oxyrhinque dont il a été question précédemment, le Peri aletheias apparaît comme un traité qui envisage tous les aspects de l'étant, depuis sa nature (physis), laquelle sous-tend

28. Pendrick 2002, p. 327 : «points forward to ».

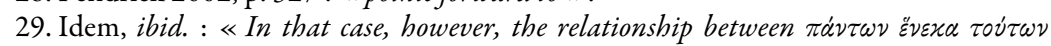
and the following ö $\tau$-clause (which also as a causal sense) is obscure. » 483a1.)

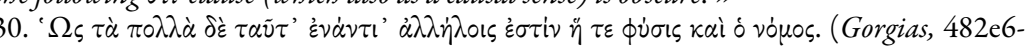


sa génération et sa corruption, jusqu'à l'organisation politique des sociétés humaines.

Parmi les fragments du traité, on range une première référence, brève mais capitale, à la conception antiphonienne de la physis, qui, pour une part, semble être une citation, au sein d'un exposé doxographique auquel Aristote procède dans sa Physique $e^{31}$. Elle est reprise par les commentateurs du Stagirite, l'un des problèmes étant alors de savoir quelles sont les limites de la citation et en conséquence à qui attribuer les notions utilisées dans ces exposés : à Antiphon lui-même ou aux discours qui rapportent ses thèses.

Cette référence/citation s'inscrit sous forme d'exemple, dans un exposé des diverses définitions qu'ont données de la physis des penseurs antérieurs. Viennent en premier ceux qui assimilent la nature à la matière et, parmi eux, d'abord ceux qui sont d'avis que la physis des étants qui sont par nature est ce qui constitue pour chacun le composant interne premier. Et d'ajouter que ce composant est par soi dépourvu de forme (arrhythmiston), ce qui désignerait la forme en question comme rhythmos ${ }^{32}$. Les exemples fournis sont alors celui du bois comme nature $\mathrm{du} \mathrm{lit}^{33}$ et du bronze comme celle de la statue ${ }^{34}$. C'est là qu'intervient Antiphon, dont on comprend qu'il a donné lui-même le premier des deux exemples puisque, ainsi que le montre la suite du texte, il l'a argumenté d'un argument par signe. Aristote rapporte l'exemple en question, tout en l'expliquant :

Antiphon en donne pour signe que, si l'on enterre un lit et que la putréfaction ait la force de faire monter une pousse, ce ne serait pas un lit mais $\mathrm{du}$ bois qui adviendrait, dans l'idée que l'on a, d'une part, ce qui appartient par accident, la disposition conventionnelle et l'art, d'autre part, cette étance qui subsiste en subissant un processus continuellement. (Phys. II, 193a1217 : trad. Stevens 1999 légèrement modifiée ${ }^{35}$.)

31. Aristote, Physique, II, 193 a 10-27.

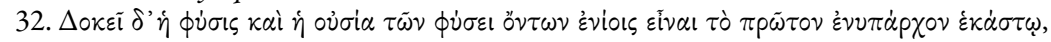

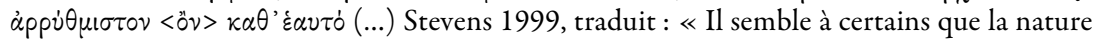
et l'étance des étants qui sont par nature est ce qui appartient en premier à chaque chose,

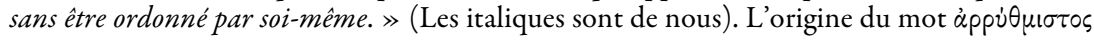
oppose Romeyer Dherbey 1995, p. 112 à Benveniste 1966, p. 332. Là où le linguiste disait qu'Aristote «forge » le mot à partir du sens de «non réduit à une forme, inorganisé», Romeyer Dherbey affirmait qu'Aristote ne s'est vraisemblablement pas donné la peine d'un néologisme pour évoquer une théorie qu'il contestait. On peut être tenté de le suivre.

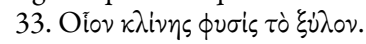

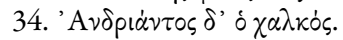

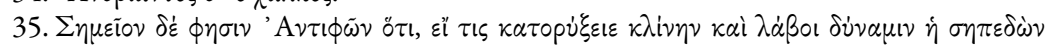

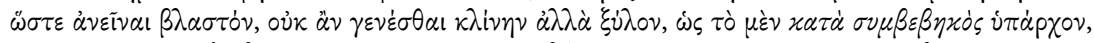

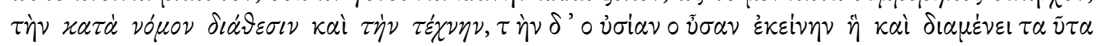
$\pi \dot{\alpha} \sigma \chi \circ v \sigma \alpha \sigma v \nu \varepsilon \chi \tilde{\omega} s$. 
À en croire Aristote, Antiphon conçoit donc le substrat interne premier comme un corps complexe, originel certes, mais non élémentaire, puisque ni le bois ni le bronze ne sont indivisibles formellement en une autre forme ou, même divisés, ne demeurent de forme semblable, comme le veut la définition aristotélicienne de l'élément ${ }^{36}$. Quoique toujours dans la rubrique de la conception de la nature comme matière, la suite du passage semble bien, en effet, donner comme différente de la conception que défend Antiphon, la thèse des physiologues qui assimilent celle-ci à l'un des quatre éléments, voire à une association de certains d'entre eux ou de tous :

Certains disent que c'est le feu qui est la nature des étants, d'autres que c'est la terre, d'autres l'air, d'autres l'eau, d'autres certains de ces <éléments>, d'autres tous. En effet, celui qui suppose que l'un de ces <éléments> est tel, qu'il y en ait un ou plusieurs, prétend que celui-ci ou ceux-ci sont la substance dans sa totalité, alors que toutes les autres choses en sont des affections, des états et des dispositions; et n'importe laquelle de ces réalités serait éternelle, car elles ne subissent aucun changement à partir d'ellesmêmes, alors que tout le reste naît et périt sans fin. (Phys. II, 193a21-28, trad. P. Pellegrin ${ }^{37}$.)

De ce début d'énumération, c'est bien Aristote qui donne l'armature logique en procédant, immédiatement après avoir énoncé la conception de type antiphonien, à une réduction à l'élémentaire d'où il fait découler l'énoncé de la deuxième conception, et cet enchaînement qui structure l'exposé aristotélicien reste extérieur, semble-t-il, à ce qu'était la thèse antiphonienne :

et si chacune de ces réalités subit la même chose par rapport à quelque chose d'autre (par exemple l'airain et l'or par rapport à l'eau, les os et le bois par rapport à la terre, et de même pour n'importe laquelle des autres réalités de ce genre), c'est cette dernière chose qui est leur nature et leur substance.

36. Car, pour Aristote, « on appelle élément ( $\sigma \tau \circ \chi \chi \varepsilon \tilde{\imath}$ V) le composant interne premier à

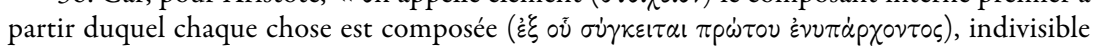
selon la forme en une autre forme (ådı éléments) se divisent, les parties sont de forme semblable : par exemple une partie de l'eau est de l'eau, mais une partie de syllabe n'est pas une syllabe $\gg($ Met. $\Delta, 3,1014 a 26-27$, trad. Duminil-Jaulin 2008, légèrement modifiée).

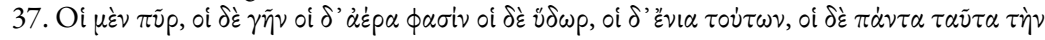

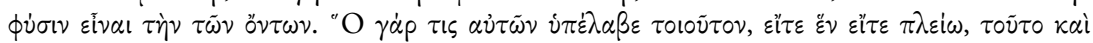

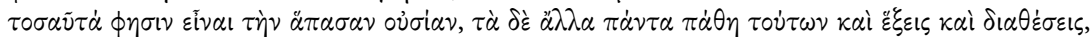

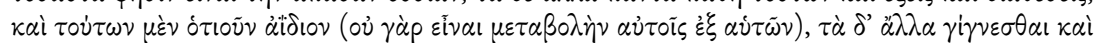
$\phi \theta \varepsilon i p \varepsilon \sigma \theta \alpha \iota \dot{\alpha} \pi \varepsilon ı \dot{\rho} \alpha \kappa \iota s$. 
C'est pourquoi, certains disent que c'est le feu... (Phys. II, 193a17-21, trad. P. Pellegrin $)^{38}$.

Une autre formulation est donnée en Met. $\Delta, 4,1014 \mathrm{~b} 26-35$, qui, en reprenant aussi les exemples attribués à Antiphon dans la Physique, vise allusivement celui-ci mais montre mieux que précédemment que la position des tenants de la nature comme matière recouvre bien deux thèses, parentes certes, mais différentes : celle du constituant interne complexe et celle du constituant élémentaire :

On appelle encore nature le fond premier dont est fait ou provient quelque objet artificiel/ naturel ${ }^{39}$, fond dépourvu de forme (arrhythmistou ontos) et incapable de subir un changement qui le fasse sortir de sa propre puissance.

Simplicius atteste aussi une telle extériorité en attribuant explicitement à Aristote la précision selon laquelle chaque «substrat prochain », ce qu'est notamment le bois dans l'exemple d'Antiphon, a lui-même un substrat, en l'occurrence la terre ou quelque autre « substrat primaire » avec lequel il est dans un rapport de forme (eidos) à substance ou nature (ousia kaiphysis) :

Puisque la forme de chaque substrat prochain a elle-même quelque substrat, Aristote ajoutait raisonnablement: « et si chacune de ces réalités subit la même chose par rapport à quelque chose d'autre $\gg^{40}$.

Mais, dans la conclusion qu'il tire, Simplicius prend bien soin de distinguer Antiphon de ceux qui sont allés jusqu'à assimiler exclusivement la nature à un substrat premier, distinction qui n'était pas aussi nette dans le texte aristotélicien :

De sorte qu'Antiphon a semblé dire en général que le substrat est nature, tandis que chacun des autres appelait aussi nature des êtres ce qu'il disait être le substrat premier, qu'il soit unique, comme Thalès le disait de l'eau,

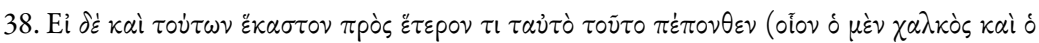

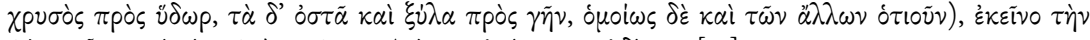

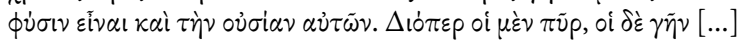

39. Selon qu'on s'en tient étroitement aux exemples d'artefacts qui seront donnés plus bas dans le texte ou qu'on met l'accent sur ce qui semble, aux yeux d'Aristote, unir la thèse du composant interne premier complexe à celle du substrat élémentaire, on lit ici $\tau \iota \tau \tilde{\omega} v \mu \grave{\eta}$

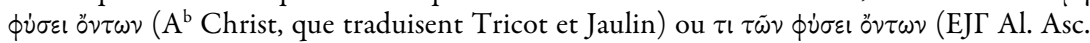
$\Phi$ Christ Ross Jaeger). Mais la nature étant, dans les deux cas, considérée comme matière, rien n'empêche qu'on parle d'êtres naturels à composant premier complexe. C'est ainsi qu'on imagine qu'Antiphon a pu, par exemple, concevoir l'homme, lequel produit en effet un homme, comme le bois, du bois.

40. Simplicius, In Aristotelis Physicorum, 274, 13-15 Diels. 
Anaximène de l'air, Héraclite du feu, qu'ils soient plusieurs, comme Parménide le disait du feu et de la terre, qu'ils soient quatre, comme le disait Empédocle. Personne ne dit qu'il s'agit de la terre seule, mais Aristote l'a posée aussi par analogie avec les autres ${ }^{41}$.

Philopon va dans le même sens que Simplicius, qui, à propos du bois dont Antiphon fait la nature du lit, parle de

quelque chose qui n'est pas simple (me haploun ti pragma), mais qui est composé (synkeitai) d'un substrat et d'une forme et qui est dans la même relation que le bois au regard du lit, le substrat et la matière du bois serait sa nature (que ce soit l'eau, la terre ou quelque chose d'autre) tandis que la forme du bois serait une affection de ce qui advient et périt ${ }^{42}$.

Le composant interne premier tel que l'aurait conçu Antiphon serait donc bien un composant non élémentaire, mais dépourvu de forme, quelque objection que ce dernier point soulève de la part d'Aristote et de ses commentateurs ${ }^{43}$.

À cela s'ajoute, toujours d'après l'exposé d'Aristote dans la Physique, que ce composant doit son statut de nature à sa capacité de demeurer continûment ${ }^{44}$. Les commentateurs d'Aristote glosent la continuité par l'iden-

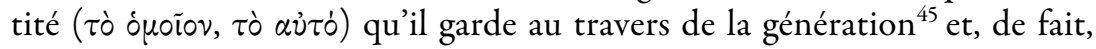

\section{Ibid. 20-26.}

42. Philopon, In Aristotelis Physica, 208, 11-16 Vitelli.

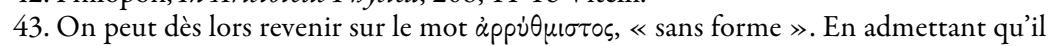
soit antiphonien, il reste alors à savoir s'il doit quelque chose à Démocrite, car il revient à

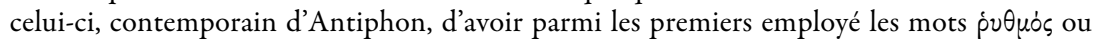
ṕofiós. A. Hourcade 2009 (chap. IX, « Antiphon démocritéen » p. 203-225, cit. p. 218219) soutient que c'est le cas, d'autant qu'on a là un composé qui, d'après elle, est assez dans la manière de l'atomiste. Toutefois on remarquera que cet emploi ne semble pas de stricte obédience car, chez Démocrite, c'est la matière que constituent les atomes et le vide qui a,

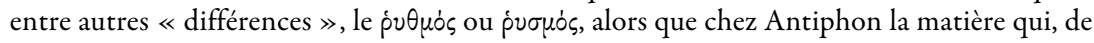
surcroît, en langage atomiste, constituerait déjà un agrégat, en est précisément dépourvue (appí $\theta \mu \tau \tau \circ \varsigma)$. Sur ce point, on fera encore appel à Aristote (Met. A, 4, 985b4-22). Celui-ci,

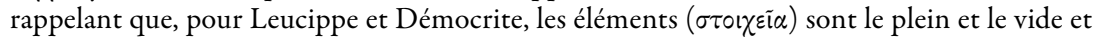
qu'ils « sont, en tant que matière, cause de ce qui est », prête aussi aux atomistes la thèse que « les différences » (des atomes individuels et de leurs relations entre eux dans le vide, $c f$. Wismann 1980, p. 69 ) sont « causes des autres choses » (les agrégats) et que ces différences

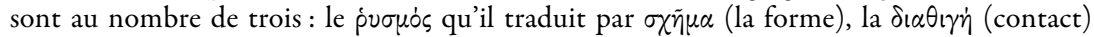
qu'il traduit par $\tau \dot{\alpha} \xi ı$ (ordre), la $\tau$ ро $\pi \dot{\eta}$ (tournure) à laquelle il donne pour équivalent $\theta \dot{\varepsilon} \sigma \iota \varsigma$ (position).

44. La définition qu'Aristote donne du « continu » (Phys. V, 227a10-17) comme ce qui se trouve dans les choses à partir desquelles quelque chose d'unique est naturellement

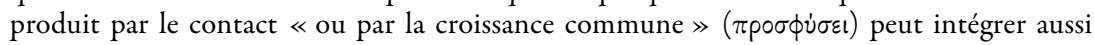
l'idée de perpétuation à travers le mouvement génératif.

45. Simplicius 283, 16-19: «Antiphon tenta de montrer la persistance de la matière

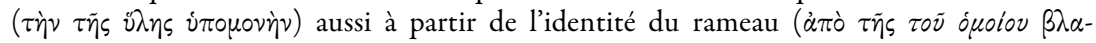


c'est ce que met d'abord en évidence l'exemple attribué à Antiphon, y compris chez Aristote. Antiphon semble en effet ne s'appuyer là que sur la constatation de la reproduction du bois en bois, lors du processus de sa génération, pour définir celui-ci comme une nature. Si aux yeux d'Aristote, le bois est en effet déjà porteur d'une forme, a fortiori le rejet qui pousse de la pièce de bois, Antiphon ne s'arrête pas à cela. Le rameau est le fruit de la puissance générative du bois en tant que bois et cela suffit à en faire d'abord du bois.

Il pourrait également dire ${ }^{46}$, tout comme Aristote, que d'un homme naît un homme, mais dans cette génération, il prétendrait ${ }^{47}$ qu'il y a permanence du composant interne complexe et non pas, comme le veut Aristote, de la forme.

Pour Antiphon, à la différence d'Aristote, l'intervention de la forme (rhythmos) $)^{48}$ est donc retardée et cantonnée au stade de la fabrication de l'artefact (le lit, la statue), lui apportant la forme comme une affection, « accidentelle », ajoute Aristote.

Mais le texte de la Physique que nous citions précédemment fait intervenir une précision capitale : Antiphon donnerait son argument par signe (semeion) que la nature du lit est le bois :

[...] dans l'idée que ( $\dot{\varsigma} \varsigma)$ ce qui est par accident, c'est la disposition selon la

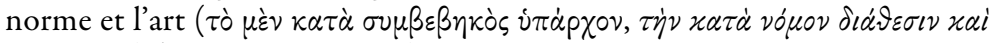
$\left.\tau \dot{\eta} \nu \tau \varepsilon \chi \nu \eta^{\prime} \nu\right)$ (Phys. I, 193a14-16).

C'est dire précisément qu'Antiphon n'aurait pas réservé l'adjonction (accidentelle) de la forme à l'art, comme le suggère l'exemple du lit, mais l'aurait aussi et plus largement attribuée à la loi/norme/coutume/, et qu'on aurait affaire, non seulement à une mise en forme technique, mais aussi ins-

$\sigma \tau \dot{\eta} \sigma \varepsilon \omega \varsigma) \gg$, par différence avec les partisans du substrat élémentaire, pour lesquels serait

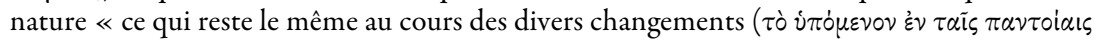

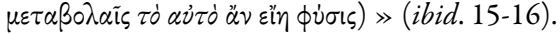

46. Et c'est ce qu'il semble faire dans les fragments de papyrus, notamment en F44a Pendrick, col. I, où l'anthropos intervient en lien avec l'évocation de la physis, par contraste avec quelqu'un qui vit sous un régime politique donné (tis politeuetai; Cassin 1995, p. 161 n. 1, traduit le verbe grec par le néologisme « celui qui citoyenne ») dans une cité (polis), domaine des nomoi et des nomima.

47. Ce que, en revanche, nous n'avons pas la chance de constater dans les textes qui nous restent de lui. Notons cependant que Philopon, commentant l'exemple antiphonien du lit, envisage aussi la question de la nature-matière $v s$ la nature-forme à propos de l'homme (anthropos), même si c'est pour soutenir, contre la théorie antiphonienne de la matière, que l'homme est forme (209, 30-210, 8 Vitelli).

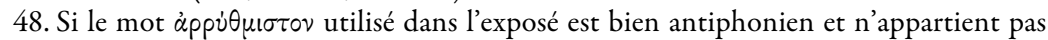
au vocabulaire aristotélicien. 
titutionnelle et, au-delà, culturelle ${ }^{49}$. Le passage a manifestement posé problème aux commentateurs. Alors que, en effet, dans le manuscrit $\mathrm{E}^{50}$, que Ross considère comme le meilleur pour la Physique d'Aristote, figure kata nomon, Simplicius signale que la leçon kata rhythmon est « la plus courante $\gg^{51}$ et Thémistius ${ }^{52}$ commente directement kata rhythmon. Mais Simplicius ${ }^{53}$ fait de ce syntagme un synonyme de kata nomon et de kata nomon, à son tour, l'équivalent $d^{\prime}$ '« établi conventionnellement par les arts ». La position de Philopon ${ }^{54}$ toutefois est plus instructive. Il atteste en effet l'existence des deux expressions kata nomon et kata rhythmon mais, dans son commentaire, les considère comme suffisamment distinctes pour se donner la peine de tenter de les concilier. Il le fait au prix de deux opérations : en premier lieu, à l'instar de Simplicius, la réduction de l'extension des normes à celle des normes de l'art (nomoi technes) c'est-à-dire à ce qui, pour une part, gouverne le choix de l'homme de l'art mais aussi, ce qui est nouveau, la définition du rhythmos comme la proportionnalité équilibrée des parts qui réalise la forme (eidos), c'est-à-dire, somme toute, comme un attribut de la forme des artefacts :

« Dans l'idée que ce qui est par accident, c'est la disposition selon la norme et l'art ». Il dit que la forme n'est pas la nature car, selon lui, sont par accident une forme et une affection du substrat et de la matière qui adviennent et qui s'en vont et qui sont tantôt l'une tantôt l'autre, tandis

49. Antiphon atteste l'usage très large de la notion de vónos dans les fragments de papyrus du Peri aletheias.

50. Manuscrit du $\mathrm{X}^{\mathrm{c}}$ siècle.

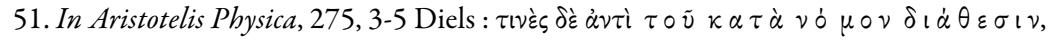

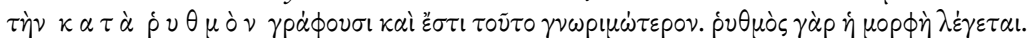

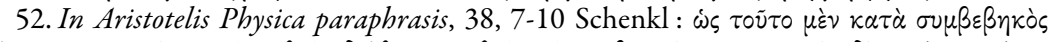

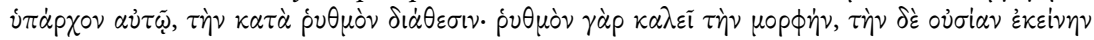

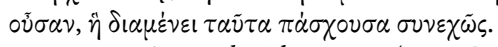

53. In Aristotelis Physica, 274, 3-5 Diels. Commentant le fait que, si l'on plantait un lit, il pousserait seulement du bois, il affirme : « Cela se produit parce que la forme est par cou-

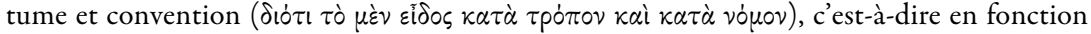

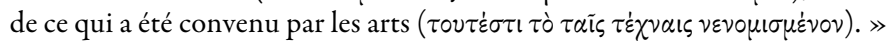

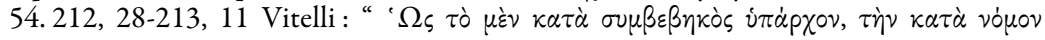

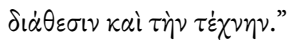

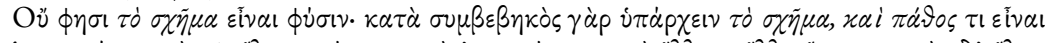

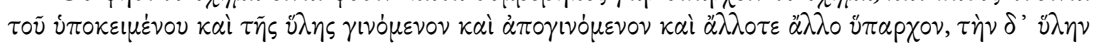

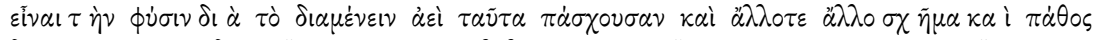

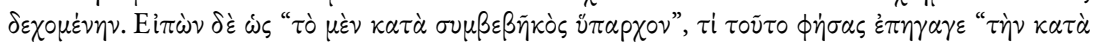

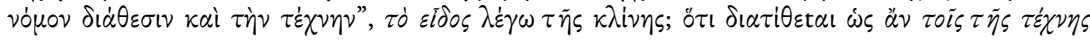

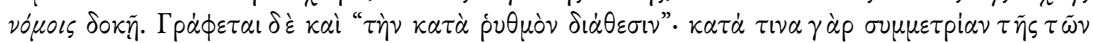

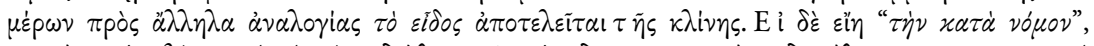

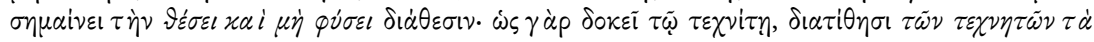

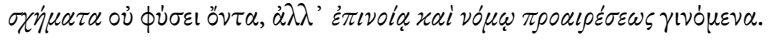


que la matière est la nature, étant donné qu'elle demeure toujours et qu'elle subit cela et qu'elle reçoit tantôt une forme et une affection, tantôt une autre. Mais, après avoir dit que la première (scil. la forme) est par accident, pourquoi, après avoir énoncé cela, a-t-il induit «la disposition selon la norme et l'art », je veux dire la forme du lit ? Parce qu'elle est disposée comme en jugent les normes de l'art. Mais on écrit aussi «la disposition selon le rhythmos » car, selon une certaine symétrie de la proportion des parties les unes par rapport aux autres, il (scil. le rhythmos) réalise la forme $\mathrm{du}$ lit. Mais à supposer que l'expression soit «la disposition selon la norme », cela signale que la disposition est par convention et non par nature. Comme en effet en juge l'homme de l'art, sont disposées les formes des artefacts qui ne sont pas selon la nature, mais selon la pensée et la norme du choix.

On peut expliquer le choix dominant en faveur de kata rhythmon au détriment de kata nomon, l'autre leçon attestée, en considérant qu'on tire ainsi le nom rhythmos de l'adjectif arrhythmiston (dépourvu de forme) par lequel Antiphon qualifie le composant premier interne de tous les êtres. Mais si, comme Philopon, on maintient à la fois la leçon kata nomon et la leçon kata rhythmon, une solution autre que la sienne s'ouvre: on peut donner à kata nomon une acception plus large dans laquelle les normes de l'art ne sont qu'une partie des normes. En ce cas, elles renverraient aussi aux coutumes et aux lois, bref à tout ce qui informe la matière du composant premier, notamment cette nature qu'est l'anthropos, capable de demeurer tel à travers la reproduction ${ }^{55}$.

L'hypothèse que nous formulons et que nous mettrons, dans un premier temps du moins, à l'épreuve de la lecture des fragments du Peri aletheias transmis par les Papyri d'Oxyrhinque consiste donc à dire que, pour Antiphon, est nature le composant interne premier des choses que révèle comme tel le processus génératif. De plus, loin d'être cause finale, comme le veut Aristote, la forme n'intervient accidentellement, à ses yeux, que dans un second temps sur ce qu'a ainsi produit la génération, que cette forme soit apportée par les règles de la production d'un artefact ou par l'application de toute autre norme $e^{56}$. En ce sens, l'être humain ne peut être

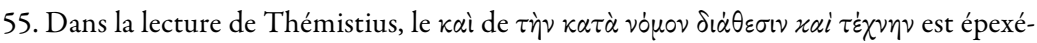
gétique ; dans la seconde, il marquerait l'association de la $\tau \dot{\varepsilon} \chi \nu \eta$ avec l'ensemble plus vaste $\mathrm{du}$ vónos.

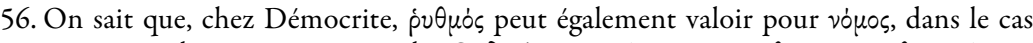

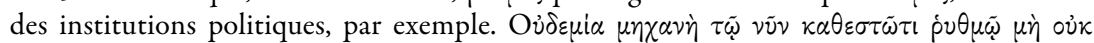
à (constitutionnelle) actuelle qui empêche que quelqu'un nuise aux magistrats (c'est le mot

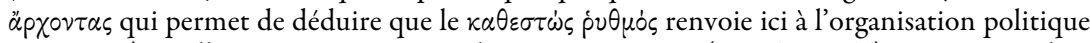
en vigueur), quelle que soit pour autant leur compétence » (DK 68 B 266). On mesure alors la force de l'interprétation que Benveniste 1976 (qui cite lui-même le précédent fragment), 
qu'une nature, dès lors qu'il se reproduit en tant qu'être humain, tandis que toutes les lois et coutumes qu'il se donne sont autant de formes qu'il s'applique, au même titre que, fabriquant ou utilisant ce qui pousse et se reproduit autour de lui, l'homme lui applique les formes diverses de ses arts.

\section{3 La nature de l'homme (anthropos) dans F44 a et b Pendrick}

Deux des trois fragments du Peri aletheias qui nous sont parvenus par les Papyri d'Oxyrhinque permettent de saisir « en extension », en quelque sorte, ce qu'Antiphon entend par nature humaine, c'est-à-dire ce qui, dans le cas de l'homme, est censé le faire advenir continûment ou à l'identique en tant qu'homme. Il s'agit de capacités (dynameis) ${ }^{57}$ et des instruments qui correspondent à celles-ci, lesquels sont énumérés au sein de développements où la nature et les normes sont mises en regard.

Dans le premier des fragments, F 44a Pendrick $=$ POxy. 1364, F1, col. II, 30-III, 18, ce sont successivement : le voir et les yeux; l'entendre et les oreilles; le dire et la langue ; l'accomplir et les mains; le déplacement et les pieds; le désirer et l'intuition, dont les lois prétendent définir l'exercice et les limites ${ }^{58}$.

Dans le deuxième, F44b Pendrick $=P O x y .1364, \mathrm{~F} 2$ et $P O x y .3647$ col. II, 15-III, 11, où Antiphon fait la liste de ce que Grecs et Barbares ont en commun au-delà des différences de leurs lois et coutumes, ce sont : respirer grâce à la bouche et aux narines; rire et pleurer, en relation, quant à

fait du lien entre $\dot{p} v \theta u$ ós et $\dot{p} \varepsilon i \bar{\nu}$, «couler». Il ne s'agit pas de dire que le nom renvoie au mouvement régulier de la mer «rythmé» par les vagues, car c'est plutôt l'écoulement d'un

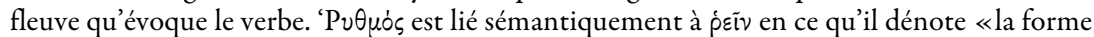
dans l'instant qu'elle est assumée», la forme «momentanée, modifiable». Quand il est associé au vónos, voire est interchangeable avec lui, ce nom pourrait donc désigner, chez Antiphon, l'arrangement accidentel et modifiable donné à la matière/nature, elle-même $\dot{\alpha} p p \dot{\theta} \theta \mu$ -

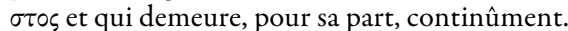

57. Toutes les reconstitutions des lignes 20-22, col. II, F44b Pendrick pour lesquelles le papyrus ne livre que $\pi[\tau \varepsilon \kappa \alpha \tau \dot{\alpha} \tau[$ [ $\nu v \alpha$ [, placent, dans l'espace après $\delta u v \alpha$ [, ou bien l'adjectif

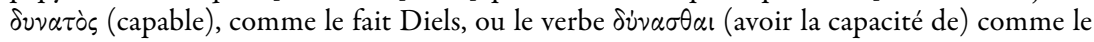

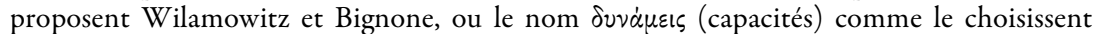
Schmidt, Theander, Bastianini \& Decleva Caizzi. Selon cette dernière leçon, qui nous semble syntaxiquement la plus aisée et, pour le sens, comme nous le verrons, la plus en cohérence avec le reste du texte, on pourrait lire les lignes 15-28 comme suit : $\sum \kappa \circ \pi \varepsilon i \nu ~ \delta \dot{\varepsilon} \pi \alpha \rho \dot{\varepsilon} \chi \varepsilon$ เ

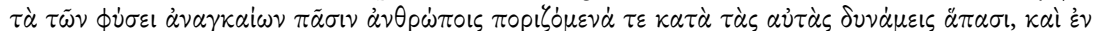

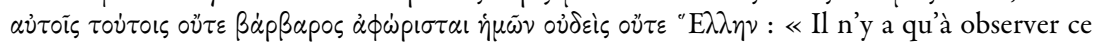
qui, pour tous les hommes, fait partie des nécessités par nature, lesquelles sont fournies à tous selon les mêmes capacités et, en ces nécessités mêmes, aucun d'entre nous n'est défini comme barbare ni comme grec. »

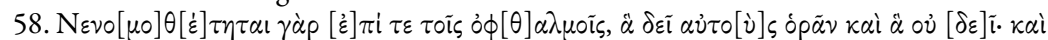

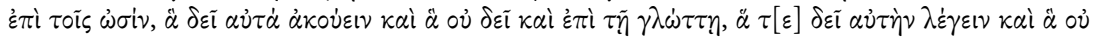

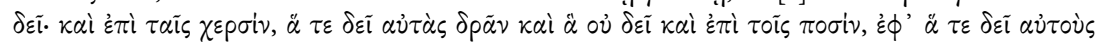

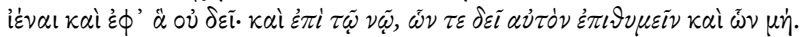


eux, avec deux états : la joie et le chagrin ; recevoir les sons articulés grâce à l'ouïe ; voir avec l'aide de la vue, grâce au rayon lumineux (qui vient de l'œil ou frappe l'œil ?) ; faire grâce aux mains et marcher grâce aux pieds ${ }^{59}$.

Ces capacités et leurs instruments ont donc en commun de posséder un même caractère fondamental et d'être dépourvus de toute spécification. Ainsi le langage est donné comme l'articulation de sons grâce à l'organe de la langue. En second lieu, pour ce qui est de la vision, Antiphon semble se référer à une conception strictement matérielle qui vaudra jusqu’à Platon inclus, et selon laquelle l'œil émet un rayon igné qui rencontre à son tour les émanations ignées des sensibles. Mais il ne sera rien dit des différentes manières qu'ont les hommes de tel ou tel groupe de s'adresser les uns aux autres, de se regarder ou d'interpréter leurs expériences visuelles dans telle ou telle circonstance. Il s'agit donc d'une humanité générique et indivise que ne spécifie aucune manière de vivre ensemble, contrairement, par exemple, à celle que postule le droit romain antérieurement au ius gentium, laquelle se compose d'hommes libres, égaux, sans différences de statuts et qui partagent dans l'indivision la terre et ses fruits ${ }^{60}$. Toutes ces qualifications relèveraient déjà aux yeux d'Antiphon d'un nomos appliqué sur le composant premier qu'est la nature humaine et qui, en tant que forme, ne pourrait que la diviser et la limiter tout en la définissant.

Au minimalisme primaire des fondements naturels correspond le dépouillement répétitif de l'expression d'Antiphon : aux mains le faire, aux pieds la marche, aux oreilles l'ouie, etc.

Ces capacités et instruments doivent en outre à leur caractère « par nature » d'avoir part à la nécessité et à l'indépassable réalité qui caractérise celle-ci. Comme nous l'avons lu précédemment en effet ${ }^{61}$, les capacités dont sont dotés les hommes actualisent chez eux ce qui appartient aux « nécessités par nature $\gg$. Ainsi est reprise l'assertion catégorique énoncée dans la colonne I du premier fragment, 1. 25-27 : « ce qui appartient à la nature est nécessaire $\gg^{62}$ et qui fait écho aux lignes $10-23$ de la colonne II du même fragment ${ }^{63}$ où, cette fois, ce qui relève de la nature est associé à la réalité/vérité (aletheia).

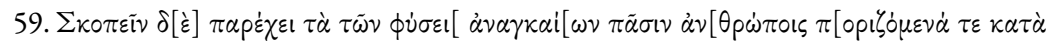

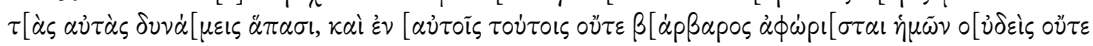

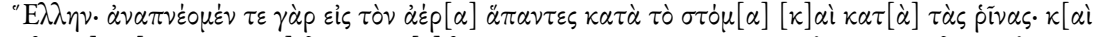

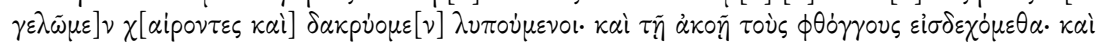

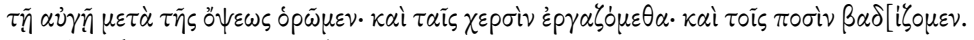

60. Thomas 2011, 21-40.

61. F44b Pendrick $=P O x y .1364$, F2 et $P O x y .3647$ col. II, 1. 15-27.

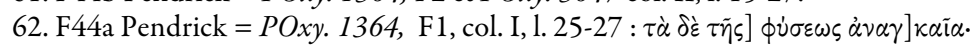

63. F44a Pendrick = POxy. 1364, F1, col. II, 1. 10-23: $\tau \tilde{\omega} \nu \delta \dot{\varepsilon} \tau \tilde{\eta} \varphi v \dot{\sigma \varepsilon l} \xi \nu \mu \varphi \dot{\tau} \tau \omega \nu \dot{\varepsilon} \dot{\alpha} \nu \tau$

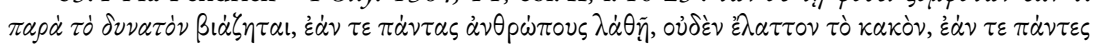

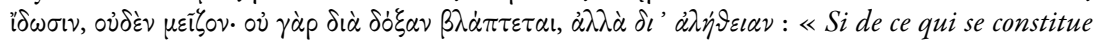


Les Tétralogies résonnent alors en écho. Ainsi, la troisième tétralogie, deuxième discours de la défense, 2, amorce, elle aussi, une énumération analogue de facultés placées sous l'égide de la nature et de sa nécessité :

À mon avis, c'est à celui qui a pris l'initiative de frapper qu'incombe l'injustice. Or il n'y a pas de vraisemblance dans les présomptions sur lesquelles se fonde l'accusateur pour prétendre que l'agresseur, c'est notre ami. Si, comme il est naturel (kata physin) aux yeux de voir et aux oreilles d'entendre, il l'était à la jeunesse de se livrer aux violences et à la vieillesse de savoir se modérer, votre jugement ne serait pas nécessaire. Leur âge suffirait à condamner les jeunes gens (Trad. L. Gernet).

\section{4 Diversité des normes (nomoi) et du juste (dikaion)}

Il n'en va pas de même de la loi. Face au socle minimal mais infrangible des données naturelles qui fondent les capacités humaines, les nomoi humains se caractérisent par les disparités qui, certes, les différencient les uns des autres, mais surtout les opposent.

Pour saisir la conflictualité qui régit les rapports entre divers ensembles de nomoi, il faut relire minutieusement le fragment POxy. 1364, F2 à la lumière du $P O x y .3647$ dont la publication relativement récente ${ }^{64}$ a révolutionné la lecture.

Après Grenfell et Hunt, pour Wilamowitz et la plupart des spécialistes qui ne connaissaient pas encore le POxy. 3647, le début du F2 du POxy. 1364 faisait l'objet de restitutions opposant les gens de haute extraction et à ceux qui ne l'étaient pas :

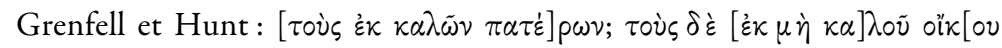

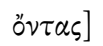

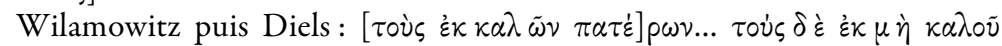
olk $[o v]$

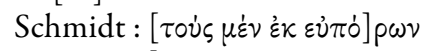

Bignone : [ $\tau \circ \dot{\varsigma} \varsigma \tau \tilde{\omega} \nu \gamma \varepsilon v \nu \alpha i \omega \nu \pi \alpha \tau \dot{\varepsilon}] \rho \omega \nu$

On déduisait de là qu'Antiphon critiquait en démocrate qu'on ménageât $\left(\dot{\varepsilon} \pi\left[\alpha i \delta \circ \nu^{\prime} \mu \varepsilon\right] \theta \alpha \tau \varepsilon\right)^{65}$ et révérât $(\kappa[\alpha i$ $\sigma \varepsilon \beta \dot{\jmath} \mu \varepsilon \theta \alpha])$ les uns mais pas les autres $^{66}$. Or la publication du fragment 3647 fait apparaître que le propos

en poussant par nature, quelque chose fait l'objet de violence à l'encontre de ses capacités, si cela se fait à l'insu de tous les hommes, le mal n'est en rien moindre et si tous le savent, en rien plus grand ; car le dommage n'est pas dû à la croyance mais à la vérité. »

64. Funghi 1984.

65. Que le $P O x y r .3647$ a permis de corriger en $\dot{\varepsilon} \pi \imath \tau \dot{\alpha} \mu \varepsilon \theta \alpha$.

66. Decleva-Caizzi 1986, signale que quelques spécialistes ont cependant, avant l'heure, hésité à adopter la lecture qui était majoritairement proposée, la jugeant hautement conjecturale: Mazzarino 1966, I, p. 609 n. 281, Moulton 1972, p. 343, Isnardi-Parente 1975, p. 17-18, Kerferd 1981, p. 154-155 et F. Decleva Caizzi elle-même. 
d'Antiphon touche, non pas aux positions sociales, mais aux disparités culturelles qui font de nous des barbares aux yeux les uns des autres. D'où des restitutions différentes dans les cinq premières lignes du fragment mais qui, cette fois, font s'opposer ceux qui sont proches de nous dans l'espace à

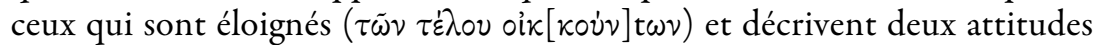
radicalement contraires envers les uns et envers les autres :

Funghi (1984) approuvée par Bastianini \& Decleva Caizzi (1989) : [ $\tau \tilde{\omega} \nu$

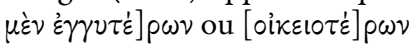

Luppe : $[\tau \tilde{\omega} \nu \pi \alpha \tau \dot{\varepsilon}] \rho \omega \nu$

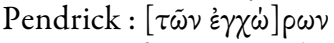

Ceux qui font partie de nos voisins/des nôtres/de nos pays, nous les connaissons et nous les respectons; mais ceux qui comptent parmi les habitants éloignés, nous ne les connaissons ni ne les respectons. En cela donc, nous en sommes venus à être des barbares ( $\beta \varepsilon \beta \alpha \rho \beta \alpha \rho \dot{\alpha} \mu \varepsilon \theta \alpha)$ les uns au regard des autres puisque, de nature ( $\phi \dot{\sigma} \sigma \varepsilon l)$ du moins, nous sommes tous en tout semblablement advenus de manière à exister, et Barbares et Grecs

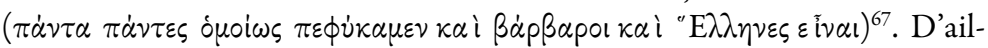
leurs, il est possible d'examiner ce qui relève des choses naturellement nécessaires $^{68}$ ( $\left.\tau \grave{\alpha} \tau \tilde{\omega} \nu \phi \dot{v} \sigma \varepsilon \iota \dot{\alpha} \nu \alpha \gamma \kappa \alpha i \omega \nu\right)$ à tous les hommes; ce qui est fourni à tous et selon les mêmes capacités $\left(\pi\right.$. $[\tau \varepsilon \kappa \alpha \tau \dot{\alpha} \tau \text { [ } \delta \nu v \alpha \text { : Pendrick })^{69}$ et aucun d'entre nous n'a été défini ni comme Barbare ni comme Grec. Car nous respirons tous dans l'air et par la bouche et par les narines et nous rions quand nous sommes joyeux et nous pleurons quand nous avons de la peine et nous recevons les sons par l'ouïe et, grâce au rayon lumineux qui accompagne la vision, nous voyons et avec les mains nous accomplissons des tâches et avec les pieds, nous marchons.

La leçon adoptée par M. S. Funghi au début de la colonne : « ceux qui sont (spatialement) plus proches » a pour elle de rappeler la conviction

67. On peut traduire aussi «nous sommes faits de manière à être et barbares et grecs » où Barbares et Grecs seraient des attributs et einai non pas le verbe être mais la copule. Cela exclut que le passage recèle ici la critique contre le Barbare comme substance, mais n'empêche pas qu'elle soit exprimée plus bas.

68. Ici l'édition princeps de Grenfell \& Hunt 1915 choisissait $\alpha 2 \alpha \gamma \kappa \alpha i \omega \nu$, tout comme plus tard Pendrick 2002, tandis que Funghi 1984 et Bastianini \& Decleva Caizzi 1989 pré-

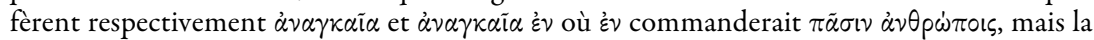
divergence est de peu d'importance.

69. Sont également sans grandes conséquences pour le sens les différentes restitutions proposées pour les lignes 20-24. Elles conduisent en effet à des sens voisins en proposant (à l'exception de celles de Schmidt et de Theander) l'idée que les hommes se voient fournir

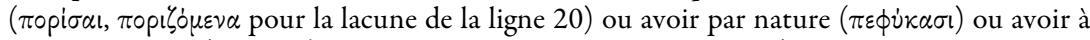

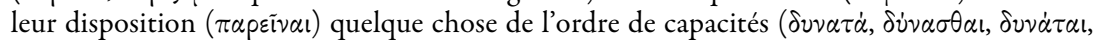
$\delta v v \dot{\alpha} \mu \varepsilon i \varsigma)$. 
qu'Hérodote $^{70}$, un quasi-contemporain d'Antiphon, prête aux Perses, à ceci près que, chez Antiphon, elle se trouverait généralisée à l'ensemble des hommes.

Quant au verbe barbarousthai, il s'agit d'un verbe rare qui signifie à l'actif « rendre barbare ». F. Decleva Caizzi ${ }^{71}$ propose de donner à la forme passive qui figure dans notre texte le sens de «adopter un comportement semblable à celui des barbares » (pour rendre, on le suppose, «avoir été rendu barbare »), cela en s'appuyant sur le vers 485 de l'Oreste d'Euripide :

Tu es devenu barbare (bebarbarosai) d'avoir vécu longtemps chez les Barbares (en Barbarois).

Je crois toutefois que cette traduction donne finalement au verbe une nuance trop active et qu'il faut plutôt opter pour une autre, « être constitué en barbare », par exemple, qui dénote plus nettement le passif car c'est bien de l'opinion dont les uns et les autres font l'objet qu'il est question, ce qui a pour effet de faire de «barbare » un attribut prédiqué d'un sujet.

Mais il faut encore ajouter que « barbare » est ici à la fois pris dans une relation réciproque (nous sommes regardés comme barbares par qui inversement sont regardés comme tels par nous : $\pi$ pòs $\alpha \lambda \lambda \dot{\eta} \lambda$ ous $\beta \varepsilon \beta \alpha \rho \beta \alpha \rho \dot{\mu} \mu \varepsilon \theta \alpha)$, et attribué à un sujet en fonction d'un relatif: la distance spatiale qui, ainsi que le dirait Aristote, admet le plus et le moins. Le raisonnement serait donc le suivant : si «barbare » est prédiqué en fonction de la distance dans l'espace, laquelle est d'un groupe par rapport à un autre, alors chaque groupe est « barbare » pour un autre, voire est « le Barbare » d'un autre et l'est plus ou moins, comme le montrent les Perses d'Hérodote. Il n'est pas sans conséquence en effet que le nom barbaroi intervienne dans le texte après le verbe bebarbarometha. De fait, Antiphon semble accomplir dans notre passage une double opération : celle de déchoir «les Barbares » du statut de substance et, en même temps, d'introduire dans le simple attribut accidentel qu'en fait le verbe bebarbarometha, quelque chose de relatif.

En quoi les nomoi que ne mentionne pas explicitement notre passage seraient-ils cependant en jeu dans l'examen critique dont la notion de bar-

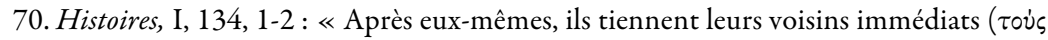
$\ddot{\alpha} \gamma \chi 1 \sigma \tau \alpha \dot{\varepsilon} \omega \nu \tau \tilde{\omega} \nu$ oik $\dot{\delta} \circ \tau \tau \alpha \varsigma)$ en très haute estime; en deuxième, ils placent ceux qui vivent un

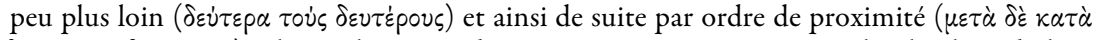

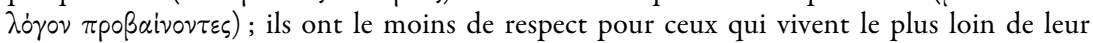
propre pays ( eux-mêmes comme de loin le meilleur peuple au monde sous tous les rapports et les autres comme décroissant graduellement en valeur de façon que ceux qui vivent le plus loin d'eux sont les pires du monde. »

71. Decleva Caizzi 1986, n. 64. 
bares/Barbares fait l'objet? On peut penser qu'ils sont introduits par la verbe sebein: respecter. Comme le remarque encore F. Decleva Caizzi ${ }^{72}$, le verbe sebein renvoie à la sphère du sacré mais celle-ci est très vaste. Pour attester cela, l'auteur cite Thucydide ${ }^{73}$ au moment où celui-ci évoque les conséquences de la peste sur les dispositions et comportements des Athéniens :

Aucune crainte des dieux, aucune loi (vónos) des hommes ne les retenait,

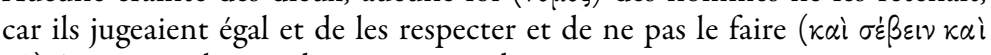
$\left.\mu \eta^{\prime}\right)$, à voir tout le monde mourir sans distinction.

C'est dire que les nomoi peuvent, aussi bien que les dieux, être objets du sebein. On peut d'autant plus penser que les normes sont impliquées ici dans la réflexion que, dans les lignes qui suivent, Antiphon fait une nouvelle énumération des nécessités naturelles proche de celle qu'il a faite auparavant $^{74}$, dans le cadre d'une mise en regard, cette fois explicite, de la nature et de la norme.

On peut donc très bien comprendre ici que la connaissance et le respect d'un groupe humain à l'égard d'un autre (et a contrario, l'ignorance et l'absence de respect), en fonction de la distance spatiale, touchent aussi bien les membres du groupe, pris ensemble ou isolément, que leurs normes et coutumes. Les distances font que les normes des uns sont connues et respectées, celles des autres non, c'est-à-dire sont « assimilées ou non à du barbare », à l'instar du groupe qui les pratique.

Les normes sont donc traversées et séparées de différences tandis que, à l'inverse, la nature est du côté de l'identité.

Mais non contentes de s'opposer de groupe humain à groupe humain, les normes sont aussi ou intimement contradictoires ou opposées entre elles dans un même groupe. C'est particulièrement évident, montre Antiphon, quand la norme a trait au juste ou à la justice (dikaion, dikaiosyne).

Quatre définitions du juste apparaissent successivement dans les fragments du Peri aletheias. La première ouvre la partie préservée ${ }^{75}$ de F44a Pendrick = POxy 1364 F1, col. I, 6-11:

72. Decleva Caizzi 1986, n. 64.

73. Thucydide, II, 53, 4.

74. Fr. 44a Pendrick = POxy. 1364, fr. 1, col. II, 30-III, 18.

75. Le texte de la colonne I est précédé d'une longue lacune dont on ne peut restituer le contenu. Il paraît tirer une conséquence, voire une conclusion, de ce qui précède ( $c f$. oũv, 1. 7). 
La justice consiste donc à ne pas transgresser les normes de la cité sous le régime de laquelle on vit $^{76}$.

Mais la cohérence de cette définition souffre dès lors qu'on envisage la justice du point de vue de sa réalisation pratique ou de son usage, car celuici fait alors intervenir des circonstances particulières : la présence ou non de témoins $^{77}$ :

Un homme mettrait donc la justice en pratique ${ }^{78}$ d'une manière au plus haut point utile pour lui ${ }^{79}$ si, en présence de témoins, il faisait grand cas des lois mais, isolé de témoins, faisait grand cas des données de la nature ; car ce qui relève des lois est appliqué sur ${ }^{80}$ tandis que ce qui relève de la nature est

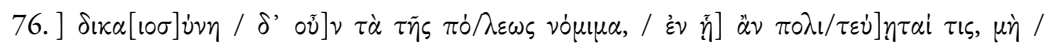
$\pi \alpha \rho] \alpha \beta \alpha i v \varepsilon เ \nu$ Pendrick.

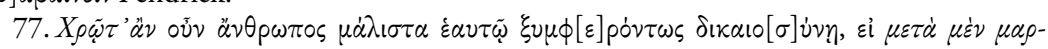

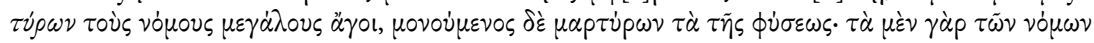

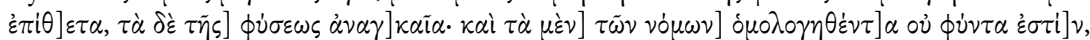

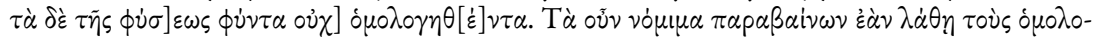

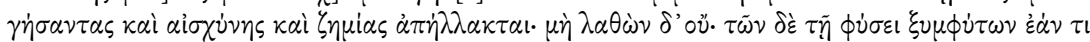

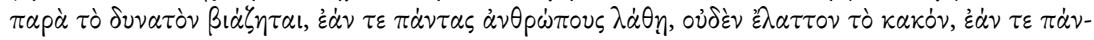

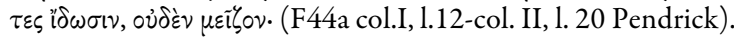

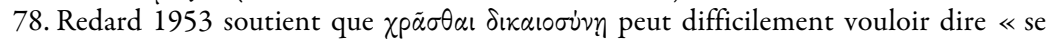
conduire justement » comme chez Hérodote II, 151, par exemple. Le sens est plutôt « pratiquer », traduction qu'adoptent Furley 1981 et Pendrick 2002, p. 322, mais qu' ils justifient d'une manière qui fait violence au texte en parlant de violation des lois en l'absence de témoins ou d'ignorance des prescriptions de la justice, là où il est question seulement « de faire grand cas » des lois en présence de témoins ou des données de la nature quand on est isolé de témoins. Certes, par la suite (col. II, 3-23), il est bien question de «transgresser » et de «faire violence », mais ce ne sont pas les normes qui sont exclusivement en jeu. Il s'agit plutôt d'envisager ce qui se passe aussi bien quand on porte atteinte aux normes qu'aux capacités qui nous viennent de la nature.

79. J'adopte pour l'instant la traduction habituelle de $\xi \nu \mu \phi \varepsilon p o ́ v \tau \omega \varsigma$. Pour le commentaire et la suggestion d'une autre traduction, voir infra.

80. $\dot{\varepsilon} \pi \imath$ - (quatre lettres) est une restitution que choisit Pendrick après Grenfell \& Hunt dans l'édition princeps. Gernet 1923, en hésitant, mais Luria 1927, 1067 et n. 3, avec assurance, préfèrent restituer $\xi \nu \nu \theta$ - tandis que Bastianini \& Decleva Caizzi jugent que l'espace de la lacune n'est pas assez grand pour cela. Pendrick, quant à lui, refuse $\xi u v \theta-$ au motif que cela anticipe sans raison ce qui est posé plus bas (1.27-31), c'est-à-dire l'idée que les prescriptions

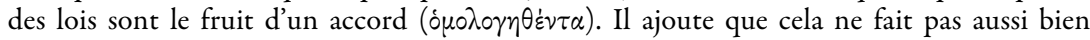
contraste avec $\dot{\alpha} \nu \alpha \gamma \kappa \alpha i \alpha$ qu' $\dot{\varepsilon} \pi \theta \dot{\varepsilon} \tau \alpha$. Pour lui, en effet, comme pour Grenfell \& Hunt, $\dot{\varepsilon} \pi \imath \dot{\theta} \tau \alpha$ ne signifierait pas «arbitraire» (willkürlich), comme le veulent Diels-Kranz, ou «occasionnel», selon Untersteiner 1962, 77, mais plutôt «imposé», «ajouté sur», surajouté à la nature, ce qui impliquerait le sens de «non nécessaire», voire $\mathrm{d}^{\prime}$ «indésirable».

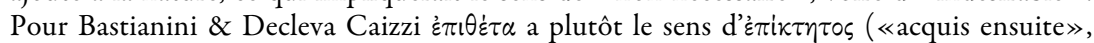

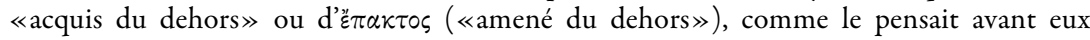
Heinimann 1945, p. 133-141. Avec la traduction par «non nécessaire », Pendrick, dont je partage par ailleurs l'analyse proche des traductions de Bastianini \& Declava Caizzi, ne 
nécessaire ; et ce qui relève des lois fait l'objet de conventions et n'est pas le fruit de la croissance vitale tandis que ce qui relève de la nature est fruit de la croissance vitale sans être objet de convention [...] Donc si l'on transgresse les lois à l'insu de ceux qui en ont convenu, on est affranchi de réprobation sociale et de châtiment pénal mais on ne l'est pas si ce n'est pas à leur insu. En revanche, si, à l'encontre de ce dont nous avons capacité ${ }^{81}$, on fait violence à quelque chose de ce que la nature fait naître tout ensemble, si c'est à l'insu de tous les hommes, le mal n'en sera pas moindre et si c'est au su de tous, en rien plus grand.

Dans le premier cas, l'impératif de non-transgression est un attribut d'essence de la dikaiosyne, dans le second il est accidentel parce que dépendant d'une donnée accidentelle. L'intervention de l'accident qu'est la présence occasionnelle de témoins fait alors affleurer une autre définition. Celle-ci est fonction du caractère propre des normes, en l'occurrence des normes civiques, au regard de celui de la physis que nous avons cru lire précédemment dans les textes concernant la physique d'Antiphon. Le nomos/rhythmos doit en effet d'être une forme accidentelle (technique, législative ou plus largement culturelle) à ce que, contrairement à la nature, il ne constitue pas le composant interne premier animé d'une puissance générative qui le reproduit à l'identique, mais quelque chose « d'apposé sur » (epitheta) celui-ci et qui peut toujours être autrement, dans le temps, sans doute, et, en tout cas, comme nous l'avons vu, dans l'espace. La nouvelle définition implicite serait donc: la justice est l'ensemble des lois que telle ou telle cité, ici ou là, applique sur la nature sans altérer la nécessité de celleci. Car, notons-le bien, il n'est pas question chez Antiphon d'un juste selon la nature ni, par conséquent, de la possibilité d'un droit naturel ${ }^{82}$.

marque pas assez, voire manque, que le mot renvoie à la physique d'Antiphon et au rapport qu'y entretiennent la matière et la forme.

81. J'ai commenté précédemment le champ lexical de dynamis dans les fragments concernant la physique d'Antiphon.

82. Voir Nesche-Hentschke 1995, p. 144. On notera aussi incidemment ce qui sépare ici Antiphon du Calliclès de Platon, auquel revient, rappelons-le, l'initiative d'introduire

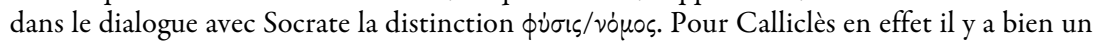

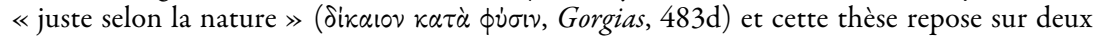
postulats qui sont radicalement différents de ceux d'Antiphon. Calliclès doit d'abord poser, contrairement à Antiphon, que la nature ne confère pas sans distinction à tous les hommes

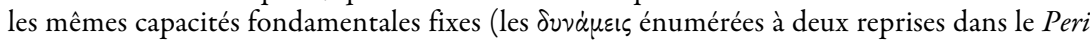
aletheias) mais à certains seulement des capacités soit supérieures, en particulier celle d'avoir

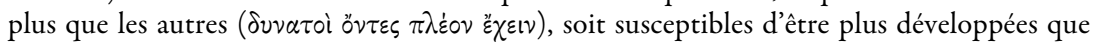

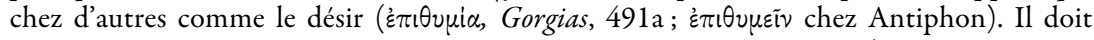
ensuite s'appuyer sur l'idée implicite que la loi est homogène à la nature (et non pas séparée d'elle par une différence ontologique comme le veut Antiphon), soit qu'elle traduise directement dans l'ordre politique de la cité (äp $\chi \varepsilon v v)$ la supériorité des plus avides et des plus désirants, soit qu'elle vienne compenser, par un régime égalitaire, la faiblesse naturelle 
Cette définition implicite vient subvertir la première que donne explicitement le texte.

La deuxième définition explicite du juste apparaît, chez Antiphon, dans F44c Pendrick $=$ POxy. 1797, col. I, 3-9 : il est juste aussi de témoigner (au tribunal) du vrai.

Témoigner les uns pour les autres de la vérité est considéré normalement comme juste et en rien moins utile pour les affaires humaines ${ }^{83}$.

Cette nouvelle définition n'est que le corollaire de celle de la justice comme non-transgression des lois : le témoin à charge s'appuie en effet sur les lois de la cité pour jouer son rôle dans un procès en illégalité. De fait, le juste ainsi redéfini souffre lui aussi de la faiblesse ontologique de la loi. Apposée sur la nature par tel ou tel groupe, celle-ci dépend de ce groupe et de ses membres pour jouer son rôle de forme. Que le contrevenant soit vu par un membre du groupe, il sera traduit en justice et fera l'objet à la fois de réprobation sociale et d'une sanction pénale. Mais il suffit que le groupe ou l'un de ses membres ne soit pas en mesure de voir pour que la forme se détache, en quelque sorte, de la matière à laquelle elle est appliquée. Le juste comme l'injuste sont fonction de l'accident de la présence tandis que, dans le domaine de la nature où il n'y a pas contingence mais nécessité, non pas lois, mais capacités issues du mouvement génératif, il n'y a absolument que

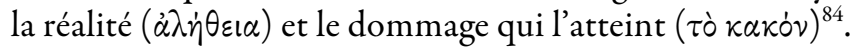

Mais si les deux premières définitions sont intrinsèquement minées par la faiblesse ontologique de la loi, la troisième définition, qui figure dans les lignes 10-19 du fragment 44c Pendrick, col. I, l'est par la contradiction qui l'oppose à un autre ordre de normes :

Celui qui fait cela (témoigner de la vérité) ne sera donc pas juste puisque ne commettre d'injustice à l'égard de personne, quand on n'est pas victime soimême d'injustice, est juste. Il est nécessaire en effet que celui qui témoigne, même s'il témoigne de la vérité, fasse malgré tout, d'une certaine manière, du tort à un autre.

À la norme civique, fondement des deux premières définitions, s'oppose en effet une norme sociale plus diffuse mais tout autant objet de convention que les précédentes : celle qui veut qu'on ne fasse pas de tort à qui ne

lamentable des autres (les $\phi \alpha \hat{\nu} \lambda \circ \mathrm{l}$ ). La loi de Calliclès n'est pas apposée sur la nature, elle en est, soit positivement soit négativement, l'expression directe. Il s'ensuit que dans l'ordre « nomique » il y aura justice à laisser jouer directement la nature et injustice à s'opposer à elle.

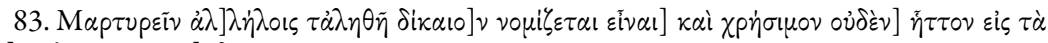
$\tau \tilde{\omega} \nu] \dot{\alpha} \nu \theta \rho \dot{\omega} \pi \omega \nu \dot{\varepsilon} \pi \mathrm{i} \tau] \eta \delta \varepsilon \dot{\nu} \mu \alpha \tau \alpha$.

84. F44a Pendrick, col. II, 1. 10-23. 
nous en pas fait d'abord, autrement dit, le juste conçu exclusivement comme abstention d'injustice à l'égard de qui ne nous a fait aucun tort. La contradiction est installée ici au prix de l'assimilation du témoignage à charge à une offense injustifiée et, cette fois, ce n'est pas sur le caractère accidentel du témoignage qu'Antiphon fait porter l'accent mais sur le «contre» du «témoigner contre» et sur la force agressive qu'il lui prête ${ }^{85}$.

Mais l'opposition entre le juste comme non-agression et le juste comme témoignage du vrai, c'est-à-dire entre une norme sociale et la norme légale explicite, s'avère réversible : celui qui défend la loi édictée en témoignant contre celui qui la transgresse va à l'encontre de l'impératif de non-agression mais, inversement, celui contre lequel un membre du groupe citoyen a témoigné conformément à la loi poursuit injustement le témoin de sa haine, parce qu'il le fait au nom de ce qui, à proprement parler, n'est pas une agression injustifiée et parce qu'il y a dans cette haine une ténacité et une durée qui excèdent l'offense supposée. On peut en effet voir affleurer ici l'idée, tout à fait explicite chez Hérodote, par exemple, selon laquelle l'excès dans la réponse à l'offense crée un nouveau déséquilibre qui relance dans l'autre sens le mécanisme de la vengeance et la soif de compensation. La première norme sociale se complète donc implicitement d'une autre comme c'était le cas pour la première norme législative au début de F44a : il est juste de répondre de façon proportionnée à une agression injustifiée.

D'un autre côté, lui-même (entendons le témoin à charge) subit une injustice de la part de celui qui a fait l'objet du témoignage à charge parce qu'il en est haï pour avoir témoigné du vrai. Et non seulement à cause de cette haine mais aussi parce qu'il doit toute sa vie se garder de celui contre lequel il a témoigné dans l'idée que, assurément, il lui est un ennemi capable de dire et de faire le mal, s'il le peut (F44c, col. I, 35-II, 12) ${ }^{86}$.

Ainsi, là où il n'y avait entre deux membres d'un même groupe civique d'autre lien que celui de l'appartenance à ce groupe, le témoignage véridique à charge dans un procès instaure entre eux un rapport d'hostilité réciproque ouvert à tous les excès. Antiphon pose donc que régler judi-

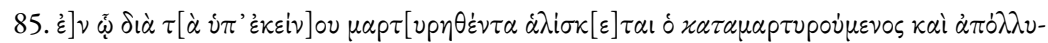

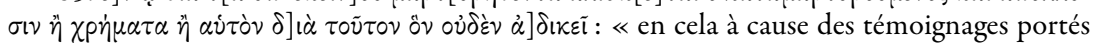
par cet homme, celui qui fait l'objet d'un témoignage à charge est condamné et perd ou ses biens ou sa vie à cause de quelqu'un à l'égard duquel il n'a commis aucune injustice » (F44c I, 1. 24-30).

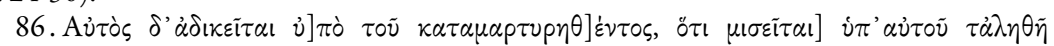

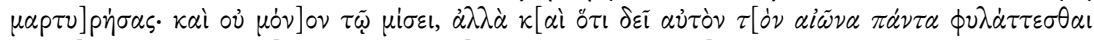

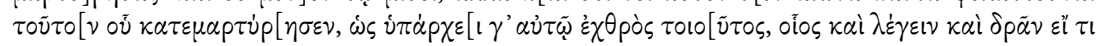

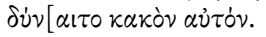


ciairement les conflits, c'est-à-dire appliquer la norme légale de non-transgression des lois, fait inévitablement ressurgir d'autres conflits au nom d'une autre espèce de norme également en vigueur dans le groupe civique.

Et ce qui vaut pour le témoignage dans un procès vaut aussi pour le juge-

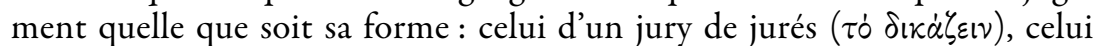

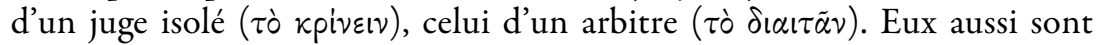
pris dans le piège de l'opposition entre norme légale et norme sociale :

Par ailleurs, et juger et décider et arbitrer ne sont à l'évidence pas justes quelle que soit l'issue car être utile aux uns nuit aux autres et, en cela, ceux qui sont aidés ne sont pas objets d'injustice mais ceux qui sont lésés le sont (F44c col. II, 25-35) ${ }^{87}$.

Ce faisant, l'incompatibilité des deux ordres de normes est alors hissée au niveau général des rapports entre l'injustice infligée et l'injustice subie :

Assurément il est évident que ce ne sont pas de petites injustices ni celles qu'il (le témoin à charge) subit ni celles qu'il inflige car il n'est pas possible qu'à la fois soient justes et de ne rien commettre d'injuste et de ne pas être soi-même objet d'injustice ${ }^{88}$ mais il est nécessaire ou que l'un des deux soit juste ou que les deux ensemble soient injustes (F44c, col. II, 12-25).

Cela vient donc poser de manière définitive que les nomoi civiques, loin de faire régner la justice dans le groupe, sont source d'injustice, non pas parce que l'injustice humaine serait un irréductible trait de nature - la nature est ici hors de cause - mais parce que ces normes sont contradictoires.

Outre la vraisemblance (Tétralogie I) ou encore le jeu entre la faute et la cause (Tétralogie II), c'est bien la conflictualité des régimes du juste qui peut nourrir les argumentaires et constituer le pivot autour duquel les plaidoyers opèrent les changements radicaux de point de vue dans le passage de l'accusation à la défense, donnant ainsi à éprouver jusqu'au vertige l'infinie réversibilité de toute position et faisant de la justice un point de fuite jamais atteint (Tétralogie III). Ce sera, par exemple, l'opposition du juste selon la loi et du juste comme réponse à une agression préalable :

Soit, répondra l'accusateur, mais la loi qui défend de tuer aussi bien justement qu'injustement te déclare passible de la peine du meurtre: car l'hom-

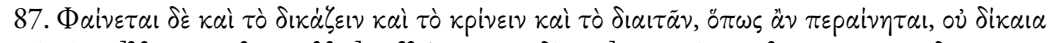

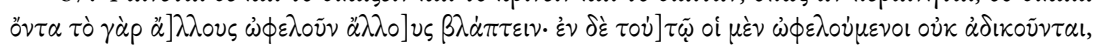

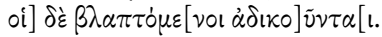

88. Ici, Antiphon s'inscrit explicitement en faux contre l'idéal socratique qui serait à la fois de ne pas subir l'injustice et de ne pas la commettre (Gorg. 469c1). 
me est mort ». Mais pour la deuxième et troisième fois, je répète que je n'ai pas tué. S'il était mort tout de suite, sous les coups, il serait mort par mon fait, quoique justement - car ceux qui ont commencé subissent justement, non pas la pareille, mais plus et pis - mais il est mort après de long jours, quand il eut été confié à un médecin incapable, victime de l'incapacité du médecin, non des coups. (III, $\beta, 3-4$.)

Admettons en effet que celui qui a frappé, en vous obligeant à recourir au médecin par suite des coups soit, plus que l'auteur immédiat de la mort, le meurtrier : alors, c'est celui qui a porté les premiers coups qui apparaît comme le meurtrier; car c'est lui qui a contraint d'abord celui qui était attaqué à frapper à son tour, ensuite la victime des coups à recourir au médecin. Et l'accusé serait traité de manière impie s'il était tenu pour meurtrier, lui qui n'a pas tué, à la place de celui qui a tué, lui qui n'a pas été l'agresseur, à la place de celui qui l'a été. (III, $\delta, 3$.) (Trad. Louis Gernet)

En revanche, cette conflictualité est systématiquement exploitée dans les discours que Thucydide, dont on fait l'élève d'Antiphon, prête aux protagonistes des événements qu'il relate, au point qu'on peut lire la Guerre $d u$ Péloponnèse comme le récit des « vicissitudes du juste », divisé en multiples acceptions qui le mettent en contradiction avec lui-même ${ }^{89}$.

L'éparpillement des nomoi, dû, à l'intérieur de chaque communauté, aux points de vue opposés adoptés sur le juste, vient alors rejoindre celui qui, pour des raisons de perception plus ou moins aiguë de l'altérité en fonction de la distance, traverse les normes des groupes envisagés les uns vis-à-vis des autres, en vertu du phénomène de «barbarisation » réciproque. Le nomos, quel que soit son champ d'application, est le lieu du multiple, du différent, voire de la contrariété, tandis que la nature en reste le socle immuable unique et unifié.

\section{5 Différence ontologique et dépendance réciproque de la nature et de la loi}

La nature et la norme, on l'a vu, ont en effet des attributs essentiels différents, et parfois contraires. Rappelons qu'à la première sont attachées la nécessité, la génération et la vérité tandis qu’à la seconde le sont la superposition, la convention et l'opinion ${ }^{90}$. Mais cette table de différences ou d'oppositions ne permet pas de conclure qu'entre les deux il y a conflit. Tout au plus y a-t-il différence ontologique.

89. Pour une plus ample démonstration qu'il serait inutile de répéter ici, voir DarboPeschanski 1987.

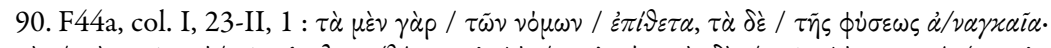

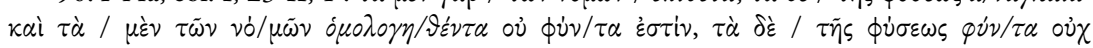

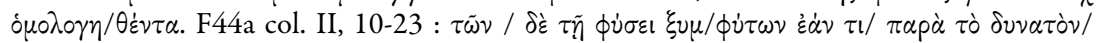

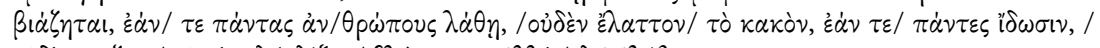

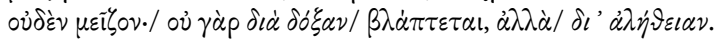


C'est ce qui justifie, non seulement de caractériser les prescriptions de la loi comme epitheta (apposées ou appliquées) sur la matière qu'est la nature, tandis que celle-ci est définie comme toujours semblable à elle-même dans le processus de génération, comme nous l'avons vu, mais aussi de comprendre que, s'agissant des capacités naturelles qui constituent l'homme, tout ce que les normes peuvent prescrire ne peut ajouter ni à leur réalité ni à leur nécessité mais seulement, en s'appliquant à elles, les limiter/définir selon des formes variables, celles d'un exercice réglé de telle ou telle manière selon les groupes sociaux considérés :

Il a en effet été établi par la loi, pour les yeux, ce qu'il faut voir et ne pas voir et pour les oreilles ce qu'il faut entendre et ce qu'il ne faut pas et pour la langue ce qu'il faut dire et ce qu'il ne faut pas et pour les mains ce qu'il faut qu'elles accomplissent et ce qu'il ne faut pas et pour les pieds vers quoi il faut qu'ils aillent et vers quoi il ne faut pas et pour l'esprit ce qu'il faut qu'il désire $e^{91}$ et ce qu'il ne faut pas ${ }^{92}$. En rien donc les choses dont les lois détournent les hommes ne sont plus amies de la nature/ plus liées à la nature ni plus propres à celle-ci que celles auxquelles elles les engagent ${ }^{93}$ (F44a, col. II, 30-III, 25).

91. L'association du voṽs avec l'appétit ( $\dot{\varepsilon} \pi(\theta) \mu \varepsilon i v)$ peut être rapprochée des conceptions présocratiques de la pensée comme processus matériel de même nature que la sensation qui, quant à elle, accueille à l'intérieur du corps la matérialité du sensible offert aux sens.

92. Beaucoup de commentateurs, préoccupés de rapprocher Antiphon le Sophiste d'Antiphon de Rhamnonte, dème où se situaient un sanctuaire de Thémis et un autre de Némésis, et où l'on rendait un culte au héros guérisseur Amphiaraos, voient dans ce passage l'énoncé d'une série d'impératifs religieux, mais le niveau de généralité auquel s'est jusqu'ici situé le texte rend cette interprétation trop restrictive, si ce n'est arbitraire.

93. Les tenants de la thèse selon laquelle, dans la phrase où intervient la skepsis, Antiphon affirme le conflit entre la loi et la nature, au lieu d'y voir, comme c'est mon cas, une manière d'annoncer l'examen critique de cette position au nom de la simple superposition de l'une sur l'autre, sont surpris de lire que ce que la loi interdit n'est pas plus aimable pour la nature que ce qu'elle autorise. On s'attendrait, dit Pendrick 2002, p. 331332, à l'affirmation inverse selon laquelle ce que la loi autorise n'est pas plus aimable que ce qu'elle interdit ou, pour le dire autrement, que ce qui est interdit n'est pas moins aimable que ce qui est encouragé (Voir Grenfell \& Hunt 1915, Furley 1981, Bastianini \& Declava Caizzi 1989). Diverses solutions textuelles sont proposées pour résoudre la difficulté : traiter la phrase comme interrogative (Theander 1920) ou comme une objection imaginaire (Barnes 1982). Gomperz 1912 propose de mettre le $\ddot{\eta}$ de III, 1.21 avant $\alpha \dot{\alpha}{ }^{\prime} \omega \tilde{\omega} v$ et donc d'inverser le comparant et le comparé, mais l'ordre des mots devient difficile à accepter. Gernet (1923) et Stenzel (1924) suggèrent qu'il y a eu inversion entre $\dot{\alpha} \phi^{\prime} \tilde{\omega} \nu \dot{\alpha} \pi \circ \tau p \dot{\pi} \pi \circ v \sigma \downarrow \nu$ et $\dot{\varepsilon} \phi^{\prime} \alpha$

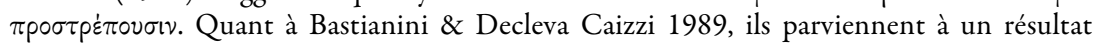

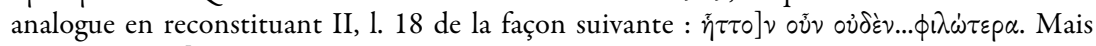
l'usage de $\tilde{\eta} \tau \tau \circ] \nu$ avec un comparatif, même s'il se rencontre parfois, reste rare. Furley 1981 et Morrison 1972 veulent éviter la difficulté en comprenant $\ddot{\eta}$ comme oủ mais les comparatifs restent alors sans complément. Heinimann 1945 et Moulton 1972 pensent que les prescriptions des lois, positives comme négatives, sont également hostiles à la nature. 
On s'arrêtera un instant sur la dernière phrase de ce passage, très compliquée par un comparatif de supériorité nié. Il s'agit ici, semble-t-il, de constater que les interdictions de la loi restreignent des/les capacités naturelles (elle fixe ce qu'il ne faut pas voir, entendre, etc.), mais que ce à quoi la loi oblige correspond aux capacités naturelles nécessaires (voir, pour les yeux, entendre, pour les oreilles, etc.). Son intervention est donc ou restrictive ou tautologique par rapport aux données de la nature, en ajoutant un devoir être à un être nécessairement, ce qui ne signifie aucunement être en opposition. Mais le texte ne s'arrête pas à ce constat. Il évalue aussi, non pas ce qui dans la loi serait hostile à la nature, comme le veut la lecture habituelle, mais ce qui, dans le champ des deux types de prescriptions normatives (ce dont elle détourne et ce à quoi elle engage), est lié/aimable (philos) et propre (oikeios) à la nature. Il s'avère alors que ce qui reste en fin de compte propre à la loi est de limiter l'exercice du donné naturel - c'est-àdire d'introduire seulement la négation propre aux bornes, laquelle ne saurait être confondue avec celle de la contrariété - sachant que lorsqu'elle ne le fait pas, elle vient seulement en redoubler la nécessité par une obligation somme toute vaine. Dès lors, ce qu'elle interdit et qui, de fait, constitue sa seule œuvre propre, ne peut se targuer de plus de « naturalité » que ce qu'elle autorise, autrement dit, son activité tautologique. Dans les deux cas, en effet, il n'y a au fondement que des œuvres liées à la nature et, pour le poser, Antiphon doit caractériser en quoi consiste exactement la sphère propre des superpositions nomiques ${ }^{94}$. Il s'agit de ce qui borne le plein exercice de ce qui est naturel par la définition apportée par telle ou telle forme, au demeurant variable et inessentielle au composant naturel fondamental. On s'étonnera cependant que le texte restreigne l'activité de la loi à la réglementation des fonctions naturelles de base. Pourquoi en effet ne pas penser qu'il pourrait y avoir du juridique pur qui ne renverrait qu'à ses propres données? Le fait est là: Antiphon n'envisage pas le corps des normes dans une éventuelle dimension «autopoiétique » et s'en tient à affirmer que, soit qu'il oblige soit qu'il interdise, il s'appuie toujours sur la nature. C'est en rester à la superposition des prescriptions de la loi à la nature. Ce faisant, c'est poser que la nature est, pour sa part, indifférente à leur forme particulière. En tant que composante matérielle pérenne et reproductible, peu lui importe qu'on lui donne pour forme celle d'un lit ou

Mais on dira, avec Pendrick 2002, que le texte grec ne permet guère cette interprétation. Quant à Diels 1916, selon lequel «ni les commandements ni les interdictions du droit sacré ou profane n'ont en eux-mêmes quoi que ce soit à voir avec la nature », il ne peut, selon moi, affirmer par là que ces commandements et interdictions n'ont rien à voir absolument avec la nature. De fait, ils s'appuient sur les données fondamentales de la nature mais le font de façons diverses.

94. D'où la formule qui surprend tant les commentateurs. 
d'un autre objet. Seul le bois pousse en effet si l'on enterre l'objet façonné. La nature (l'homme, en l'occurrence) peut donc se voir appliquée n'importe quelle disposition conventionnelle sans en être affectée comme matière.

D'où encore le constat formulé très clairement plus loin (F44a col. IV, 1-7):

Quant aux choses utiles ${ }^{95}$, celles qui sont là pour avoir été posées du fait des lois sont des liens de la nature, tandis que celles qui l'ont été par la nature sont libres ${ }^{96}$.

Mais cela ne signifie pas qu'il ne faille pas nécessairement une forme. L'étant humain n'a pas besoin d'être le membre de telle communauté politique ou de telle société précises mais il est nécessaire qu'il soit en quelque manière le membre d'un groupe qui a et se donne des lois. Seulement la nature est indifférente à cette manière.

Il y a donc plus que la différence ontologique qui place la norme du côté du superposé et du délimitant. La norme en effet ne peut exister sans le support matériel de la nature. C'est ce que révèle l'analyse du début préservé du fr. 44a col. I, 6-23 :

La justice consiste donc à ne pas transgresser les normes de la cité dans le régime politique de laquelle on vit. Un homme mettrait donc la justice en pratique d'une manière au plus haut point utile/constitutive pour lui si, en présence de témoins, il faisait grand cas des lois mais, isolé de témoins, faisait grand cas des données de la nature.

Le cadre de référence posé ici est celui d'une cité quelconque, sans considération de son régime, et s'impose en vertu de l'idée que ce sont les normes, quelles qu'elles soient, qui définissent la citép ${ }^{67}$. Le cas considéré à l'intérieur de ce cadre est, au même degré de généralité, celui d'un membre quelconque d'une cité exerçant les prérogatives qui lui sont accordées dans cette cité en vertu des lois, quelles qu'elles soient, de celles-ci. Le troisième pilier de cette armature civique épurée est la justice, elle-même fonction des normes de la cité considérée. Quant aux témoins dont il est très vite question, ce sont, comme le texte le montre abondamment par la suite et comme nous avons pu le constater à maintes reprises auparavant, les protagonistes de l'institution judiciaire civique du procès.

95. Si l'on s'en tient encore une fois, pour l'instant, à la traduction habituelle.

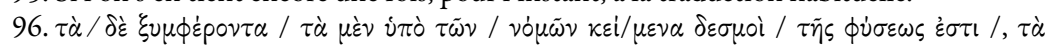

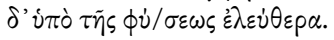

97. C'est ce qui fait dire à Cassin 1995, p. 162, que, pour Antiphon, la cité est définie par du «nomique $»$. 
C'est de la même justice (le mot dikaiosyne revient ligne 16) que le texte envisage ensuite la mise en pratique. On reste donc dans le même cadre civique mais avec un changement de point de vue qui engage dans un processus de découverte. Dès lors qu'il s'agit d'exercer de la justice, en effet, il suffit que celui qui est défini par la norme du groupe civique et vit sous son égide se trouve seul (monoumenos), en l'absence d'éventuels témoins, (autrement dit sans la présence du groupe en la personne de concitoyens susceptibles de témoigner dans une procédure judiciaire civique) pour qu'il se saisisse de fait en étant naturel humain (anthropos). Le membre de la communauté civique est la forme apposée sur l'être humain et cette nature ne peut apparaître que lorsque l'isolement soustrait quelqu'un au groupe, c'est-à-dire au principe organisateur de celui-ci qu'est la loi ${ }^{98}$.

Mais la nature elle-même peut-elle rester sans forme? L'homme (anthropos) peut-il vivre absolument seul, sans le groupe «nomique » et réduit à l'exercice des quelques capacités élémentaires que nous avons précédemment énumérées ? On pense ici à ce que dira plus tard Aristote ${ }^{99}$ de l'homme sans cité, l'apolis, semblable à un pion isolé dans un jeu qui, on le suppose, repose sur la combinaison de plusieurs autres pions ${ }^{100}$ comme le groupe social repose sur la combinaison de plusieurs membres. Il ne s'agit pas alors d'un homme mais d'un être d'une autre nature : bête ou dieu. Pour Antiphon également l'homme est toujours déjà social mais, pour lui, c'est par une sorte de passage occasionnel à la limite qu'est l'isolement au cœur du groupe qu'il peut faire l'expérience de sa constitution naturelle, tout comme, épistémologiquement, c'est par l'opération de soustraction de la forme au support matériel de celle-ci qu'il en acquiert la connaissance et la fait connaître.

Antiphon met encore en évidence, semble-t-il, un tel primat existentiel de la loi lorsque qu'il envisage le cas limite suivant (F44a, col. IV, 32-VI, 27) : à supposer qu'on se conforme rigoureusement à la norme sociale de non-agression et qu'on refuse d'entrer dans les mécanismes judiciaires du témoignage à charge, on n'échappe pas à l'injustice pour autant. Cependant, bien qu'il constate avec la plus grande dureté que, tout compte fait, la norme ne protège en aucune manière ceux qui vivent sous son règne et

98. Cassin 1995, n. 1, p. 171 : « La nature (chez Antiphon) est ce qui reste au creux du politique (...). Pour Antiphon, comme pour Protagoras et pour Gorgias, nous sommes d'abord des êtres logico-politiques et ensuite seulement, à nouveau ou autrement, des animaux physiques. »

99. Aristote, Pol. 1, 2, 1253a1-7.

100. Ce qu'on traduit le plus souvent par « jeu de tric-trac », plus qu'un « jeu de dés » serait peut-être un « jeu de plateau », comme le jeu de dames, qui repose sur les positions respectives des pions et leurs relations. Sur la portée du passage pour la philosophie politique d'Aristote, voir Labarrière (à paraître). 
réduit tout le monde à être châtié, Antiphon s'arrête malgré tout à ce constat, sans aller jusqu'à prôner de sortir du règne « nomique » et d'opérer une sorte de « retour à la nature ».

Chez ceux qui, s'ils ont pâti, se défendent sans prendre eux-mêmes l'initiative d'agir et ceux qui traitent bien leurs parents même si ces derniers se conduisent mal envers eux et ceux qui laissent les autres prêter serment contre eux mais ne prêtent pas eux-mêmes serment à charge et dans tout ce qui a été dit auparavant, on pourrait trouver que bien des choses sont hostiles à la nature; du moins y a-t-il là et de quoi souffrir davantage alors que moins est possible et de quoi prendre moins de plaisir alors que plus est possible, c'est-à-dire de quoi être maltraité alors qu'il est possible de ne pas l'être. Si donc à ceux qui se soumettent à une telle situation une assistance venait des lois tandis que pour ceux qui ne s'y soumettent pas mais s'y opposent il en venait une perte, il ne serait pas sans utilité d'obéir aux lois. Mais en fait, à ceux qui se soumettent à ce genre d'exigences, le juste issu de la loi n'est manifestement pas capable de venir en aide. En tout cas, ce qu'il laisse à celui qui subit, c'est de subir et à celui qui commet une exaction, de la commettre; et là il n'empêche pas celui qui subit de subir, non plus que celui qui porte atteinte de porter atteinte, et rien de ce qui apporte un châtiment n'est plus propre à celui qui a subi qu'à celui qui a agi.

Tout au plus peut-on lire ici un pessimisme fondamental à l'égard de l'efficacité de la loi au regard de la justice mais ce serait prolonger indûment le propos du texte que de lui faire dire que les normes montreraient ici leur hostilité à l'égard de la nature, et qu'il serait non seulement souhaitable mais encore possible de s'affranchir de celles-ci. On sera en effet sensible, dans le passage, au potentiel an heuroi, 1.14-15 et au ge (1.15):

et dans tout ce qui vient d'être dit on pourrait trouver que bien des choses sont hostiles à la nature; du moins y a-t-il là et de quoi souffrir davantage alors que moins est possible et de quoi prendre moins de plaisir alors que plus est possible, c'est-à-dire d'être maltraité alors qu'il est possible de ne pas l'être (1. 14-23).

Cette formulation vient donner à l'hostilité la valeur d'une idée qu'on pourrait éventuellement avoir mais à laquelle il convient d'apporter une restriction et qu'il faut reformuler. Il s'agit en effet de s'arrêter à constater que, dans le groupe social ou civique, même si l'on essaie de se garder des effets des lois, celles-ci accroissent la peine sans augmenter le plaisir. Or, comme le nous verrons plus loin, la nature ne s'identifie pas forcément au plaisir. Antiphon n'engage donc pas à s'affranchir des lois pour « jouir sans entraves $\gg$.

Ainsi, dire, comme le fait Antiphon, que la nécessité est l'un des attributs de la nature et non de la norme (laquelle, rappelons-le, peut toujours 
être autrement, et entre les groupes et dans chaque groupe, puisque chacun peut «citoyenner» de diverses manières) ne signifie pas que la dépendance réciproque de la nature et de la loi ne soit pas, quant à elle, nécessaire. Elle l'est. De même en effet que tout constituant premier n'échappe pas à la forme technique, légale, ou culturelle, de même dans le cas précis du constituant homme, il n'y a pour ainsi dire pas moyen d'éviter l'emprise du nomos, quelles qu'en soient les espèces. Le défaire de cette nécessité soit ne dure qu'un temps fugitif, celui de la brève expérience de l'isolement, soit ne peut être que l'objet d'une opération intellectuelle abstraite de soustraction.

Un mode d'expression très caractéristique d'Antiphon, dans le Peri aletheias mais aussi dans les plaidoyers, vient corroborer ce qui précède. Il s'agit de l'usage répété, fût-ce parfois au prix de la clarté, comme nous l'avons vu plus haut, de formules de comparaisons niées ${ }^{101}$. Certes le premier mouvement porte à songer à la formule sceptique (phone skeptike) ou mallon / ouden mallon par laquelle, comme le rapporte Sextus Empiricus ${ }^{102}$, on entend implicitement «pas plus ceci que cela et inversement» ou «pour quelle raison ceci plutôt que cela ? » et qui renvoie à l'affection (pathos) qu'on éprouve quand, du fait de la force égale des choses opposées (ton antikeimenon pragmaton), on est conduit à l'équilibre (arrepsia), c'està-dire à l'impossibilité de donner son assentiment à aucun des deux côtés. Mais pas plus que la skepsis d'Antiphon ne peut s'interpréter comme celle des sceptiques, ainsi que nous l'avons vu, ses comparatifs niés ne visent à créer l'équilibre de l'indécidable à partir d'une opposition, à égalité, de la nature et de la loi, en l'occurrence. Ils ont plutôt pour fonction d'affirmer, dogmatiquement, diraient des sceptiques, la dépendance (le comparatif) déséquilibrée (la négation) de la nature humaine et de la norme selon des points de vue variés. Nous avons remarqué plus haut, en effet, que cette dépendance peut être envisagée du point de vue de la «naturalité »: les choses qu'interdit la loi qui limitent ou lient la nature ne sont pas plus « naturelles » que celles qu'elle prescrit positivement et qui en redoublent la nécessité : les deux ont la nature pour support. Ailleurs, c'est la question

101. Tétralogie I, $\Gamma, 3:$ : Enfin, pour ceux qui étaient moins en danger, je ne vois pas comment ils auraient prémédité le meurtre plutôt que ceux qui avaient davantage à redouter. »Ibid. 7 : «Si la crainte du soupçon avait suffi pour le détourner de l'attentat alors qu'il était exposé aux plus grands périls, personne, à ce compte, n'aurait prémédité le meurtre: car tous ceux qui couraient moins de danger, redoutant le soupçon plus que le danger, auraient été encore moins disposés que lui à l'attentat. »Ibid. $\Delta, 4:$ « L'infortune même qui leur sert à me calomnier, je demande qu'elle se change en bonne fortune et j'estime qu'il vaudra mieux pour vous m'estimer heureux pour m'avoir absous que de me prendre en pitié après m'avoir condamné. » (Trad. Louis Gernet.)

102. Sextus Empiricus, HP I, 188-190. 
de l'atteinte portée aux normes et de celle qui frappe la nature qui est envisagée :

Donc, si l'on transgresse les normes à l'insu de ceux qui en ont convenu, on est affranchi de honte et de châtiment pénal mais on ne l'est pas, si ce n'est pas à leur insu. En revanche, si, à l'encontre du potentiel naturel, on fait violence à l'une des dispositions que la nature fait croître ensemble, si c'est à l'insu de tous les êtres humains, le mal n'en sera pas moindre et, si c'est au su de tous, il n'en sera en rien plus grand. Car ce n'est pas à cause de l'opinion/du jugement qu'elle subit la violence mais à cause de la réalité. (F44a, col. II, 3-23.)

On pourrait résumer ainsi le propos: dans un cas, la sanction (honte sociale, peine judiciaire) peut tomber ou ne pas tomber (selon que l'infraction est perpétrée totalement en cachette ou pas), dans l'autre le mal intervient quoi qu'il en soit de sa visibilité et de la connaissance qu'autrui en a. De surcroît, on notera les contrastes lexicaux : d'un côté, transgression des normes (parabainein), de l'autre, violence (biazein) à l'égard de ce que la nature engendre et des capacités qu'elle donne; d'un côté, des sanctions sociales ou pénales, de l'autre, le mal (kakon) pris absolument ; d'un côté, le ou les membres de groupes civiques, de l'autre, les hommes ou étants naturels. On notera cependant que les comparaisons concernent uniquement le mal qui est fait à la nature. Celui-ci n'admet aucun degré et affecte tous les humains ${ }^{103}$, en tant que natures, indépendamment de la connaissance qu'ils en auraient ou n'en auraient pas. Il s'agit donc moins ici de décliner l'idée de l'hostilité des normes vis-à-vis de la nature que de préparer une nouvelle disparité ontologique qui marque encore une fois la faiblesse des normes et leur dépendance vis-à-vis de la nature (1. 21-23): celle de l'opinion/jugement (doxa) et de la réalité/vérité (aletheia). Les atteintes à la loi demandent la présence d'une instance de jugement (celle d'un témoin) pour prendre corps et être sanctionnées; les atteintes à la nature ont une réalité objective que ne peut forger ni moduler aucun jugement. On retrouve donc ici, à travers l'énoncé de cette nouvelle différence ontologique, le caractère second et superposé de la loi par rapport à la nature : la loi est de l'ordre du jugement porté par un citoyen présent aux faits délictueux, mais dès lors que la nature est en jeu, il ne reste au jugement - si d'aventure jugement il doit y avoir - qu’à se conformer à l'intangible réalité de celle-ci et par là à être vrai. En toute rigueur ontologique toutefois, elle peut se passer de ce jugement. $\mu \varepsilon \tilde{\zeta}, \mathrm{o \nu}$.

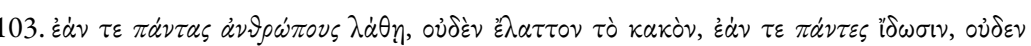


Mais la dépendance peut s'inverser, en changeant de nature. La totalité de l'humanité qui est évoquée à deux reprises dans le texte n'est en effet à proprement parler que théorique. D'une part ne sont concrètement impliqués que les membres de tel ou tel groupe social « nomique » (étant donné que, pour Antiphon, l'homme est de fait toujours déjà en société, comme nous l'avons vu); d'autre part, et de ce fait, c'est grâce à leur opinion de vérité, c'est-à-dire à l'exercice de leur jugement, que ces hommes socialisés peuvent atteindre la réalité de la nature et par là en exhiber les attributs essentiels $^{104}$.

\section{6 Confirmation par l'homonoia}

Il est une notion dont nous savons qu'elle a fait l'objet d'un traité attribué à Antiphon ${ }^{105}$ et qui illustre parfaitement l'interdépendance déséquilibrée de la nature et des normes : l'homonoia ${ }^{106}$. Celle-ci n'est jamais définie explicitement dans les fragments qui nous restent mais l'un d'entre eux, cité par Stobée ${ }^{107}$, peut au moins en donner une idée. L’homonoia semble s'accomplir dans des relations sociales, c'est-à-dire dans la sphère des nomo $i$, mais prend son origine et trouve son fondement dans la nature.

Soit le mariage. C'est dans le cours de la vie biologique que naît à un moment donné le désir d'une union et d'une femme ${ }^{108}$, autant dire ici de s'unir à une femme ${ }^{109}$. Or, rappelons que le désir figure dans la liste qu'An-

104. Comme le note Cassin 1995, p. 170-171, n. 1, la distinction que fait Parménide entre $\dot{\alpha} \lambda \dot{\eta} \theta \varepsilon i \alpha$ et $\delta \dot{\delta} \xi \alpha$ semble reconduite par Antiphon, mais au prix d'un renversement qui, au lieu de poser d'emblée l'être où tout se déploie, donne à la $\delta \dot{c} \xi \alpha$ en œuvre dans le politique la fonction de trouver à sa racine, et en quelque sorte en creux, la vérité de la nature.

105. Selon Philostrate, Vies des sophistes, I, XV, $4=2,17$ Kayser $=$ T2(b) Pendrick, à côté de ses discours judiciaires, Antiphon aurait composé des discours «sophistiques » dont le Peri homonoias serait le plus marqué en la matière. L'auteur use alors de critères majoritairement formels (des sentences brillantes, un style solennel, fleuri de termes poétiques et d'une débordante ampleur), encore qu'il crédite le traité d'une certaine portée philosophique.

106. Pour Hourcade 2001, p. 96-97, il faut faire conjointement appel au Peri homonoias et au Peri aletheias pour comprendre la perspective d'Antiphon, un avis que nous partageons, mais elle affirme aussitôt que, au-delà des différences qu'il y a entre un traité plutôt descriptif et un autre plus interprétatif et plus systématique, un point commun demeure : la dénonciation (implicite dans un cas, ouverte dans l'autre) du nomos humain comme violence faite à la nature, et sur ce point nous ne la suivons pas.

107. IV, XXII, $66=$ DK 87 B $49=$ F 49 Pendrick.

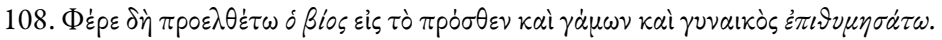

109. Comme le dit Vernant 1974, p. 59 et 62, songeons que, même dans l'Athènes classique, la cité démocratique tente de privilégier certaines formes d'union par rapport à d'autres afin de perpétuer le corps de citoyens et une bonne circulation des biens, «le mariage n'est pas une institution parfaitement définie» mais constitue plutôt un «état de fait» : le synoikein, mot qui recouvre pour la femme le fait de partager à la fois la demeure et le lit de l'époux. 
tiphon dresse des capacités naturelles, c'est-à-dire réelles et nécessaires, et a pour instrument le nous (l'intuition). L'union avec une femme, soutenue par le désir de faire couple ${ }^{110}$ représente donc la face naturelle de l'homonoia, mot qu'Antiphon, comme à son habitude ${ }^{111}$, invite à comprendre au plus près de sa composition comme « similitude d'intuition ou d'activité du nous $\gg$.

Mais, comme nous le verrons encore une fois plus bas, ce qui est naturel n'est pas obligatoirement plaisant. Ainsi l'union durable avec une femme revient en quelque sorte pour un homme à ne plus être seul dans « son corps », conçu ici sans distinction d'avec l'esprit, comme ce qui fait de l'anthropos une entité vivante. Il doit s'occuper de la santé et de la subsistance d'un autre corps (et s'il vient des enfants, d'autres corps) que le sien. L'une de ses capacités naturelles fondamentales, le désir, le conduit au souci, voire à la douleur, d'avoir à perdre son autonomie, son intégrité monadique, ellemême naturelle.

Quoi de plus agréable pour un homme qu'une femme selon son désir ? Quoi de plus doux, surtout quand on est jeune? Mais dans cette même situation où se trouve le plaisir se trouve aussi, pas très loin, ce qui apporte la douleur. Car les plaisirs ne vont pas seuls : les chagrins et les peines leur font cortège [...] Moi, si j'avais à m'occuper d'un autre être, semblable à moi, je ne pourrais plus vivre, tant sont nombreuses les préoccupations qui m'assaillent pour la santé de mon être, pour trouver de quoi vivre chaque jour [...] Que serais-je alors, si j'avais à m'occuper de quelqu'un d'autre et que j'en aie autant de souci que pour moi ? Il est donc clair qu'une femme ne cause à un homme, lorsqu'elle est chère à son cœur, non moins de tendres plaisirs et de soucis qu'il ne s'en cause à lui-même : il y a à se soucier de la santé de deux êtres à trouver de quoi vivre (...) Là-dessus, que surviennent des enfants : alors c'est une nuée de soucis à tout propos, l'élan de la jeunesse n'habite plus dans la pensée et l'on commence à changer de visage ${ }^{112}$.

110. Il n'en va pas différemment pour Aristote qui, dans Les Politiques, fait du couple homme-femme l'une des communautés (koinoniai) naturelles de base.

111. Cf. infra.

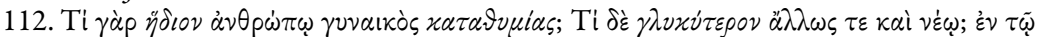

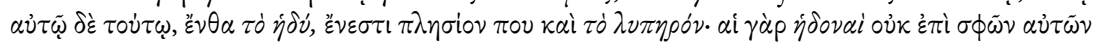

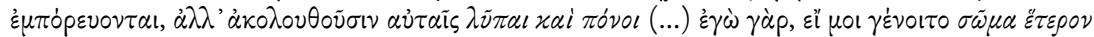

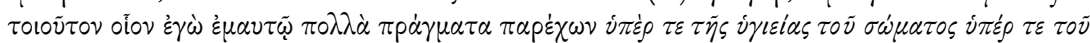

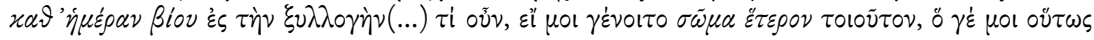

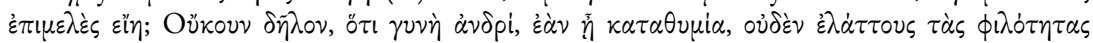

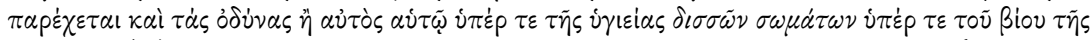

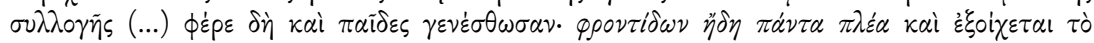

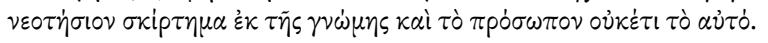


L'homonoia traduit donc, dans la nature, la tension entre l'un et le multiple, tension sur laquelle s'articule celle du plaisir et de la peine, certes, mais tension nécessaire, comme tout ce qui est naturel aux yeux d'Antiphon, puisque l'homme, en tant qu'étant naturel, ne peut se reproduire à l'identique, c'est-à-dire en tant qu'homme, sans la reproduction sexuée.

Mais, dans le cas du mariage, l'homonoia a également des prolongements sociaux qui l'inscrivent dans l'ordre de la norme. Le mariage s'accompagne en effet du versement d'une dot au futur tuteur de la femme que deviendra son mari, après qu'elle aura quitté la puissance d'un homme de sa famille (père, oncle ou fils), et sanctionne ainsi l'alliance de deux maisons. Mais la cohabitation est-elle rompue? La femme réintègre alors avec sa dot son groupe familial d'origine et retrouve son premier tuteur ${ }^{113}$. Les deux maisons rompent alors leurs liens :

Divorcer est chose pénible ; c'est se rendre ses affins ennemis, alors qu'on ne faisait qu’une seule pensée, qu'un seul souffle ${ }^{114}$, alors qu'on leur avait donné son estime et qu'on avait obtenu la leur en retour ${ }^{115}$.

On peut penser, à l'inverse, que les références à des pensées et à des paroles partagées sans disparité, ainsi qu'à une réciprocité d'estime, sont une manière de gloser l'idée de similitude comprise dans l'homonoia à laquelle le départ de l'épouse a mis fin entre deux groupes familiaux. Il y a donc une homonoia «nomique ${ }^{116}$ qui, elle aussi, comporte ses douleurs et ses plaisirs. Elle doit en effet être maintenue avec effort tant que dure l'union puisque l'époux doit, outre se préoccuper de la santé et de la subsistance de deux êtres, et non plus de lui seul, leur assurer aussi « la considération, la modestie prudente, l'honneur et la bonne réputation ${ }^{117} \gg$.

113. De même que la pompe, procession par laquelle on conduit la femme chez son époux et nouveau tuteur (kyrios), est le rite social par lequel s'officialise l'union de deux familles, de même l'ekpompe qui, au sens propre, désigne la sortie de la maison de l'époux, sanctionne aux yeux de tous la rupture de cette union.

114. On peut voir ici une manière de renvoyer à l'idée que le souffle porte la parole, comme l'indique le participe pepnymenos qui, dans l'Iliade et dans l'Odyssée, désigne souvent le personnage qui s'apprête à parler.

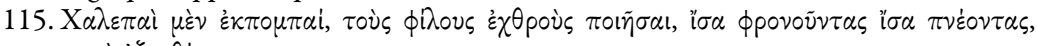

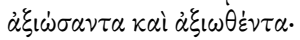

116. On notera incidemment que le Peri homonoias permet ici de saisir, mieux que ce n'était le cas dans le Peri aletheias, que la normativité de la vie du groupe s'accompagne aussi d'une psychologie sociale impliquant la pensée, la parole et l'évaluation des uns par les autres.

117. On retrouve ici ce qui différencie le nomos de la nature, caractérisée quant à elle par

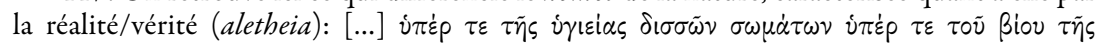

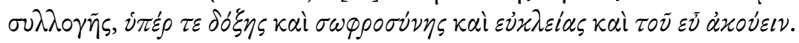


Ainsi, à la différence de la justice qui s'inscrit exclusivement dans l'ordre des normes du groupe, l'homonoia, de par son fondement naturel et son inscription dérivée dans la sphère sociale, donne à comprendre une nouvelle figure du rapport de dépendance ontologique de la norme vis-à-vis de la nature.

\section{Le xympheron, l'utile et l'agréable}

\section{1 Sens du xympheron}

Dans un passage du Peri aletheias (F44a, col. III, 25-IV, 22 $2^{118}$ ), la confrontation de la nature et des normes, tout en ayant recours à une nouvelle formule de comparaison niée, s'approfondit encore en faisant intervenir les couples xympheron/me xympheron; algynein ou lypein /euphrainein ou hedein; oninenai ou ophelein/blaptein:

Le vivre, en effet, relève de la nature, ainsi que le mourir et le vivre, lui, vient de ce qui est constitutif et le mourir de ce qui n'est pas constitutif. Quant aux choses constitutives, celles qui sont là pour avoir été posées par les lois sont des liens de la nature, tandis que celles qui le sont par la nature sont libres. Assurément, les choses qui causent de la peine ne sont pas, à bien raisonner en tout cas, plus utiles à la nature que celles qui réjouissent. Assurément, les choses qui affligent ne sauraient non plus être plus constitutives que celles qui font plaisir. Il faut en effet que les choses réellement constitutives ne nuisent pas mais soient utiles.

L'un des intérêts majeurs de ce développement réside dans la différence qu'il permet de faire entre le xympheron, d'une part, l'utile ou l'avantageux, de l'autre. Si l'on opte en effet pour l'équivalence des deux, la dernière phrase du texte énonce simplement une redondance, dont on ne voit ni l'intérêt ni, à proprement parler, le sens, en soi ou dans le mouvement du texte :

Il faut en effet que les choses réellement utiles/avantageuses ne nuisent pas mais soient utiles.

Mais la première phrase nous invite à une autre traduction. Si un étant naturel (le bois, un homme, etc.), se constitue dans la génération et se défait dans la destruction et la mort, comme le suggère la première phrase du passage, il conviendrait alors de revenir au sens premier du mot xympheron:

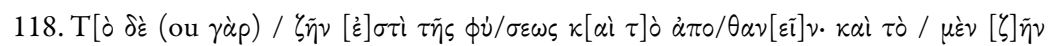

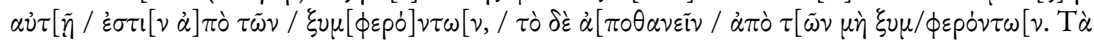

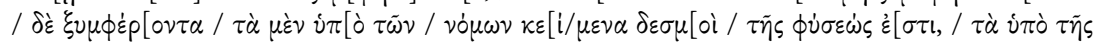

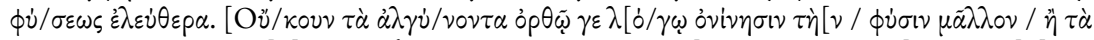

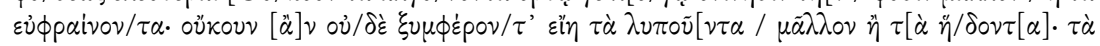
$\gamma \dot{\alpha} \rho \tau \tilde{\omega} / \dot{\alpha} \lambda \eta[\theta \varepsilon] \tilde{\imath} \xi u \mu \phi \dot{\varepsilon} / \rho[o v \tau] \alpha \circ \dot{\nu} \beta \lambda \dot{\alpha} / \pi[\tau \varepsilon l] \nu \delta \varepsilon \tilde{\imath} \dot{\alpha} \lambda\left[\lambda{ }^{\prime} \dot{\omega} / \phi[\varepsilon] \lambda \varepsilon i \tilde{\nu}\right.$. 
« qui apporte ensemble », «qui réunit », « qui rassemble», ce que je propose de traduire par « constituant » ou « constitutif». Il s'agit en effet de rendre au mieux l'idée d'un mouvement qui porte (en l'occurrence celui de la génération qui fait advenir à l'existence) contenue dans phero et celle d'ensemble impliquée par syn- (en l'occurrence l'ensemble constitué par tel ou tel étant naturel particulier et complexe : le bois, l'homme, etc.), sans pour autant se déterminer sur ce qui est rassemblé, ni sur les détails du processus de rassemblement.

Nous ne sommes pas loin en cela de la suggestion de Socrate dans le Cratyle (417c) selon laquelle il faut associer les $\sigma u \mu \phi \dot{s} p o v \tau \alpha$ (sympheronta) aux $\sigma \dot{v} \mu \phi o p \alpha^{119} \pi \rho \dot{\alpha} \gamma \mu \alpha \tau \alpha$ (symphora pragmata), en faisant ressortir par là leur lien commun avec la фopd் (phora), le transport qui, en même temps (hama), associe (meta) $)^{120}$.

Nous sommes également conduits à songer sur ce point au dialogue entre Socrate et Thrasymaque dans la République (I, 336-347). Là, en effet, tandis que son interlocuteur ramène le sympheron à l'utile/avantageux sous les espèces de l'ophelimon et du kerdaleon, Socrate suggère de celui-ci une définition proche de celle du Cratyle. Ainsi, dans le second moment de la discussion (à partir de 343b), après que Socrate a renversé en son contraire la thèse de son interlocuteur en se faisant un levier de la comparaison que celui-ci a introduite entre le gouvernant et l'artisan (demiourgos) et a conclu que le juste (dikaion) est que le gouvernant commande pour le sympheron du gouverné, reste encore dans l'ombre ce qu'il faut dès lors entendre par sympheron.

Thrasymaque donne sa réponse au moment où il vient d'aggraver sa thèse en deux temps : d'une part, les plus forts - politiquement - visent leur intérêt - qu'il appelle toujours sympheron - et les gouvernés sont des naïs qui appellent justice le dommage (blabe) qu'il y a pour eux à travailler pour «l'intérêt » des plus forts; d'autre part, extrême pointe de la démarche qui a mené à mettre l'injustice du côté du plus fort $(343 \mathrm{~d} 2$ 3), l'homme juste a partout le dessous vis-à-vis de l'injuste. Pour l'interlocuteur de Socrate, il n'y a aucune différence entre le sympheron et ce qui est matériellement avantageux. Ainsi, de même que le berger ou le bouvier, et au-delà d'eux, les maîtres dont ils sont les esclaves, élèvent leurs troupeaux pour en tirer profit : ophelein (343b8), de même les gouvernants dans leurs rapports avec les gouvernés. Soient, par ailleurs, diverses relations qui se nouent dans la cité: celles des contrats (symbolaia), celles des

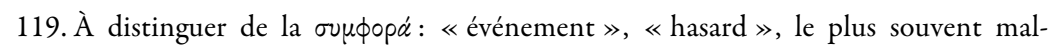
heureux, accentué sur la pénultième (oxyton).

120. En l'occurrence, c'est celui qui associe l'âme aux choses et qui, par là, rapproche le sympheron de la science (episteme). 
contributeurs à l'impôt (eisphora) ou des bénéficiaires des distributions d'excédents auxquelles la cité procède à l'occasion ou encore celles qui, dans le domaine des charges politiques (archai), associent magistrats et administrés ou enfin celles, extrêmes, du tyran avec tous ceux qui sont sous sa domination, dans tout ces cas donc, l'avantage (sympherein, 344a2) de l'injuste consiste à «avoir plus» (pleon echein, 343d4-5), à «payer moins » (elatton espherein, $343 \mathrm{~d} 8$ ), à «accumuler beaucoup de biens » (polla kerdainein, 343e1), à «acquérir plus» (pleonektein, 344a1), et, s'agissant du tyran, à s'emparer du bien d'autrui » (aphaireisthai tallotria), non pas en détail mais tout d'un coup, par la fraude et la violence (344a78).

C'est cette assimilation du sympheron avec l'ophelimon, le kerdaleon, voire le lysiteloun (344c8), entendus ici comme « ce qui est matériellement profitable », que Socrate défait, d'une part en utilisant l'assimilation du gouvernement (arche) à un art (techne) que lui a proposée Thrasymaque et, d'autre part, en faisant du seul art du mercenaire la techne dont la fonction propre (dynamis) est de procurer un gain ou salaire (misthos) à celui qui l'exerce. Ainsi le rapport gouvernant/gouverné se trouve libéré de son lien avec la recherche du profit matériel chez le plus puissant pour devenir une association (syn-) dans laquelle le gouvernant apporte le fruit (porizein) de sa compétence au gouverné qui en a besoin, comme le médecin apporte la santé à son patient ou l'architecte la maison à qui veut s'abriter. La nouvelle acception du sympheron ainsi suggérée fait donc advenir entre eux une communauté dont le fondement n'est pas l'exploitation de l'un par l'autre, et «l'intérêt personnel » de l'un au détriment de celui de l'autre. Pour ce qui est de la République, la voie est ainsi ouverte à l'enquête sur le fondement de cette communauté, en l'occurrence la justice sur laquelle repose la constitution de chacun comme de la cité par le bon partage et la bonne hiérarchie des fonctions. Cela nécessitait de nettoyer la notion de sympheron d'une acception particulière et restrictive pour revenir à ses sèmes fondamentaux dégagés dans le Cratyle.

Le parti pris, en quelque sorte analytique, adopté dans notre lecture du xympheron d'Antiphon a pour lui, semble-t-il, d'être en accord avec ce qui apparaît par ailleurs comme « la manière » de celui-ci : il aime à donner sens à toutes les composantes d'un mot, notamment en jouant sur les préfixes ou les dérivations rares et par là saillantes. On pensera à l'opposition a po-trepein (détourner) par opposition à pro-trepein (tourner vers, engager à) (F44a, col. III, 22-24); au sens très littéral que semble prendre epi- (sur) dans epitheta (placé sur) (F44a, col. I, 25); ou encore à l'effet de contraste ménagé en F44a, col. II, 4-23 entre le couple «à l'insu de » (lathe $(i))$ / «au su de » (me lathon) dans la transgression des lois et l'a- 
letheia (la réalité/le non-caché121) de la nature qui rend absolu et indifférent à la présence ou l'absence de témoins le mal qu'on peut lui faire. On ajoutera à cette liste l'articulation théorique à laquelle il est procédé entre le suffixe homoios et le nom nous dans le concept d'homonoia. Dans la rubrique des dérivations, on placera le très remarquable verbe monoo (F44a col. I, 20): « réduire à l'unité », « isoler » qui, chargé d'une tonalité épique ${ }^{122}$, fait contraste avec le vocabulaire très sobrement classique d'Antiphon et qui attire fortement l'attention sur monos: «seul», « sans la compagnie d'autrui », donc sur l'idée de mise à l'écart du groupe, fondamentale pour l'interprétation de ce passage en ce qu'elle ne doit pas être confondue avec on ne sait quel repliement sur une individualité intimement préoccupée d'intérêt personnel.

Ce passage précédemment évoqué vient en outre conforter l'interprétation du début de F44a proposée plus haut. L'anthropos (l'étant naturel humain) trouve la meilleure manière de se constituer (malista beauto(i) xympherontos: « de la manière la plus constitutive pour lui »), en l'occurrence d'advenir en tant que tel à l'expérience et à la connaissance du membre de la communauté civique (celui qui pratique la justice comme nontransgression des lois de la cité), si ce membre se trouve isolé du groupe « nomique » et, par là, en mesure de prendre en considération les données de la nature, alors que, dans ce groupe, ce sont les lois qu'il regarde comme importantes. Certes, la distance que la présence d'autrui permet de mettre entre le membre du groupe et l'homme, lesquels, comme le texte le montre ensuite, n'ont pas le même statut ontologique, peut induire une position de retrait et de calcul d'intérêt vis-à-vis de la loi. Mais là n'est pas le propos immédiat du texte, comme plus tard, dans la République, le sens particulier et indûment généralisé que Thrasymaque donne au sympheron ne peut, ainsi que le montre Socrate, en constituer sans contradiction le sens premier.

Le second intérêt capital du passage tient à ce que, via le couple xympheron/me xympheron, il distingue l'utile/nuisible du couple réjouissant/affligeant (euphrainon/algynon) ou pénible/agréable (lypoun/hedon). Dire, en effet, que ce qui afflige ne saurait être plus constitutif que ce qui fait plaisir ne revient pas à dissocier le radicalement xympheron de la peine pour lui associer exclusivement le plaisir ni à le couper du plaisir pour lui associer exclusivement la peine, mais bien à mettre celui-ci à distance aussi bien de la peine que du plaisir. Ce qui est constitutif (le xympheron) est utile seulement en ce qu'il fait l'intégrité d'un étant mais ne lui garantit pas que, constitué comme tel et avant que la mort ne le défasse, il ne vive que dans le plaisir.

121. Un Antiphon heideggerien avant la lettre, somme toute.

122. Il est employé dans l'Odyssée. 
On notera en effet qu'il y a deux espèces de xympheron, l'un selon la nature, l'autre selon la loi. Le premier assure la constitution et la dissociation des étants naturels et, parmi eux, de l'homme; l'autre assure celles des membres des groupes sociaux et politiques. Mais le parallélisme entre les deux n'est pas parfait, toujours en raison de la différence ontologique de la nature et de la loi. Tandis que la loi, apposée qu'elle est sur la nature, ou bien en limite les données ou bien, quand elle autorise, se contente de laisser jouer les capacités naturelles, c'est-à-dire, pour une part, constitue le membre du groupe social par restriction, la nature, quant à elle, constitue pleinement l'homme (étant naturel) en tant qu'homme.

Mais cette distinction ne recouvre pas celle de la peine et du plaisir. On peut fort bien imaginer, par exemple, que la loi édicte des récompenses, des gratifications, des manifestations festives incontestablement plaisantes (l'homonoia quand elle devient fait social a aussi ses joies) et que, inversement, vivre, c'est-à-dire le xympheron selon la nature, puisse n'être pas exempt de peine, ni de chagrin, ni de souffrance. En revanche, même pour éprouver de la peine, il faut que le vivant soit constitué. Le constitutif a plutôt partie liée avec l'utile mais seulement en ce que le nuisible défait, qu'on se place dans l'ordre «nomique » de l'appartenance sociale (on peut frapper un citoyen d'atimie ou rejeter un de ses membres hors du groupe, comme Philoctète par exemple, et ainsi porter atteinte plus ou moins durablement à sa qualité civique ou sociale, voire à ses chances de survie) ou qu'on soit dans celui de la nature, en amoindrissant plus ou moins radicalement la vitalité d'un étant.

\section{2 Le soi-même selon Antiphon}

La tradition selon laquelle Antiphon aurait pour propos de déclarer que, au nom de «l'intérêt personnel », il faut laisser parler la nature contre la loi est longue et puissante ${ }^{123}$. Jonathan Barnes soutient de façon convaincante que notre auteur constate sans engager à quoi que ce soit. C'est bien ce que nous avons pu vérifier. Toutefois le problème est de savoir ce qu'il constate.

Il s'agirait seulement, selon J. Barnes, de faire apparaître en sociologue que, dans une cité (Athènes peut-être), les lois se contredisent et sont impuissantes à ménager l'intérêt de chacun et, comme la majorité des spécialistes, de traduire F44a, col. I, 12-21 en introduisant l'idée d'avantage rapportée à soi-même :

123. Sahlins 2008 montre combien la conception occidentale qui ramène la nature humaine à son avidité native est une donnée culturelle occidentale parmi d'autres mais qui se prétend universelle et que, de longue date, nous sommes enclins à admettre sans réticence depuis nos sociétés d'économie capitaliste en arguant d'un « selfish system ». 
Hence a man will deal with justice in the way most avantageous to himself (beauto(i)) if in the presence of witnesses he holds the law high, and when isolated from witnesses the dictates of nature ${ }^{124}$.

Ce soi-même qui apparaîtrait dans le heauto(i) de la ligne 12 n'est jamais questionné. Mais tandis que J. Barnes ne tire pas de conclusions hasardeuses d'une telle traduction, d'autres, tels les aventuriers d'on ne sait quelle arche perdue ${ }^{125}$, tombent dans ce gouffre et glissent vertigineusement au long d'interminables boyaux sinueux et pentus (d'avantages en intérêts personnels, d'intérêts personnels en individu, d'individu en individu $v s$ les autres, d'intériorité) jusqu'à être déposés, émerveillés, dans une caverne où ils trouvent les trésors convoités : la loi qui contraint $v s$ la nature avec ses désirs et ses plaisirs qui poussent à une réalisation de soi prédatrice et égoïste.

À ce compte, et sans s'arrêter à la place qu'Antiphon donne explicitement «au soi-même », on en déduit que son propos ne saurait être autre que moral. Je crois, pour ma part, que comme les sophistes plus ou moins contemporains, Antiphon mène une réflexion ontologique qu'il complète, nous en sommes d'accord, par un constat sociologique, mais qu'il ne se livre à aucune spéculation sur le sacrifice que la loi et avec elle le groupe social imposerait à un «individu » doté d'une « intériorité » dans laquelle celui-ci enfouirait à regret le sens aigu de son « intérêt personnel » sans cesse menacé par les autres.

À montrer plutôt que le xympheron est défini, non pas comme l'utile ou l'avantageux, mais comme «ce qui constitue »(syn-phero), on peut constater que le propos de ces textes est de faire apparaître que le membre d'une communauté civique « constitué » par les lois, se trouvera constitué en étant naturel (anthropos) dès lors qu'il aura l'occasion de ne plus être sous le regard de ses concitoyens. C'est dire que l'autos ne renvoie pas ici à une intériorité psychologiquement intéressée d'elle-même. Au sein d'une réflexion ontologique selon laquelle la loi se trouve « apposée sur » (epitheta) la nature, tandis que celle-ci est le composant interne reproductible, nécessaire et réel de tout ce qui existe, l'idée serait plutôt que, la condition

124. Barnes 1982, p. 509.

125. Le prix revient ici à M. Nill, 1985. Quant à A. Hourcade 2001 en se fondant sur des «analogies» entre Démocrite et Antiphon ainsi que sur des phénomènes « d'influence », elle avance que l'individu antiphonien est à l'atome démocritéen ce que la cité est à l'agrégat. Cette position qui repose, sur des bases parfois fragiles (certaines données lexicales : $c f$ n. 7, le rapport entre les sens et gnome) a ceci de séduisant qu'elle permet dans un premier temps d'éloigner d'une conception psychologique moderne de l'individu. Toutefois l'auteur en vient à conclure sur la morale d'Antiphon, sur le fait qu'il pense l'éthique et le politique du point de vue de l'individu, et sur l'impossible coïncidence des autres avec soimême, ce qui paraît ramener à cette perspective. 
de membre d'un groupe régi par des normes étant toujours déjà en place, l'anthropos (l'étant naturel) ne peut apparaître que comme ce qui reste, lorsque, grâce à un isolement temporaire (monoumenos), la forme apposée qui est civique est, de ce fait, temporairement «levée ». Le « soi-même » ici n'est donc pas une substance, mais seulement l'interface, sans aucune autonomie, entre la forme «nomique » qu'est le membre de la communauté civique et le composant naturel qu'est l'anthropos. 


\section{BIBLIOGRAPHIE}

Barnes, J. 1982 : The Presocratic Philosophers. 2, Empedocles to Democritus, Londres, ${ }^{2} 1982$ (The Arguments of the Philosophers).

Bastianini, G. \& F. DeCleva CaIzZI 1989 : «Antipho », dans Corpus dei papiri filosofici greci et latini $(C P F)$ : testi e lessico nei papiri di cultura greca e latina. Parte 1.1, Autori noti. 1.1*, A-C, Florence, 1989 (Studi e Testi per il Corpus dei Papiri Filosofici), p. 176-222 ( $\left.\mathrm{n}^{\circ} 17\right)$.

Benveniste, É. 1976: «La notion de "rythme" dans son expression linguistique », Problèmes de linguistique générale, Paris, 1966 (Bibliothèque des sciences humaines), p. 327-335.

CASSIN, B. 1995 : L'effet sophistique, Paris, 1995 (NRF essais).

COUlOUbARITSIS, L. 1990 : «La problématique sceptique d'un impensé : $\dot{\eta} \sigma \kappa \varepsilon \dot{\psi} \psi \varsigma$ », dans A. J. Voelke (éd.), Le scepticisme antique: perspectives historiques et systématiques: actes $d u$ Colloque international sur le scepticisme antique, Université de Lausanne, $1^{\mathrm{er}}$ 3 juin 1988, Genève-Lausanne-Neuchâtel, 1990 (Cahiers de la Revue de Théologie et de Philosophie, 15), p. 9-28.

Darbo-PeSChANSKI, C. 1987 : «Thucydide : historien, juge », Métis, 2.1 (1987), p. 109140.

Decleva Caizzi, F. 1985 : «Ricerche su Antifonte : a proposito di POxy. 1364, fr. 1 », dans M. Capasso, F. de Martino, P. Rosati (éd.), Studi di filosofia preplatonica, Naples, 1985, p. 191-208.

- 1986: «Il nuovo papiro di Antifonte, POxy LII, 3647 » dans F. Adorno, F. Decleva Caizzi, F. Lasserre \& F. Vendruscolo (éd.), Protagora, Antifonte, Posidonio, Aristotele: saggi su frammenti inediti e nuove testimonianze da papiri, Florence, 1986 (Accademia toscana di scienze e lettere La Colombaria. Studi, 83 ; Studi e testi per il Corpus dei papiri filosofici greci e latini, 2), p. 61-69.

DesClos, M.-L. 2009: «Antiphon de Rhamnonte (environ 480-411 av. J.-C.) », dans J. F. Pradeau (dir.), Les sophistes: fragments et témoignages. 1, De Protagoras à Critias, Paris, 2009 (GF, 1338), p. 161-325.

Diels, H. 1916 : «Ein neues Fragment aus Antiphons Buch Über die Wahreit (Oxyrh. Pap. XI N. 1364) », Sitzungsberichte der Berliner Akademie, 38 (1916), p. 931-936.

Dillon, J. 1984 : « Euripides and Antiphon on Nomos and Physis: Some Remarks », dans

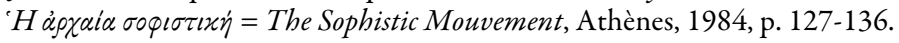

Duminil, M.-P. \& A. JAULIN 2008: Aristote, Métaphysique, introduction, traduction, notes, bibliographie et index, Paris, 2008 (GF 1347).

FARENGA, V. 2006 : Citizen and Self in Ancient Greece: Individuals performing Justice and the Law, Cambridge, 2006.

FunghI, M. S. 1984 : POxy. 3647, dans H.M. Cockle, The Oxhyrynchus Papyri, 52, edited with translation and notes by -, Londres, 1984 (Graeco-Roman Memoirs, 72), p. $1-5$.

FurLeY, D. J. 1981 : « Antiphon's Case Against Justice », dans G. B. Kerferd (éd.), The Sophists and their Legacy: Proceedings of the Fourth International Colloquium on Ancient Philosophy Held in Cooperation with Projektgruppe Altertumswissenschaften der Thyssenstiftung at Bad Homburg, 29th August - 1st September 1979, Wiesbaden, 1981 (Hermes Einzelschriften, 44), p. 81-91.

Gagarin, M. 2002: Antiphon the Athenian: Oratory, Law and Justice in the Age of the Sophists, Austin, Tex., 2002.

GERnET, L. 1923 : Antiphon, Discours, suivis des Fragments d'Antiphon le Sophiste, texte établi et traduit par -, Paris, 1923 (Collection des Universités de France).

GOMPERZ, H. 1912 : Sophistik und Rhetorik, Leipzig-Berlin, 1912. 
Heinimann, F. 1945 : Nomos und Physis: Herkunft und Bedeutung einer Antithese im griechischen Denken des 5. Jahrhunderts, Bâle, 1945 (Schweizerische Beiträge zur Altertumswissenschaft, 1). [Réimpr. Darmstadt, Wissenschaftliche Buchgesellschaft, 1972.]

Hourcade, A. 2001: Antiphon d'Athènes: une pensée de l'individu, Bruxelles, 2001 (Figures illustres).

- 2009 : Atomisme et sophistique : la tradition abdéritaine, Bruxelles, 2009 (Cahiers de philosophie ancienne, 21).

HUMBeRT, J. 1972 : Syntaxe grecque, Paris, ${ }^{3} 1972$ (Tradition de l'humanisme, 8).

ISNARDI PARENTE, M. 1975: «Egalitarismo democratico nella sofistica ? » Rivista Critica della Storia della Filosofia, 30 (1975), p. 3-26.

Kerferd, G. B. 1956-1957: « The Moral and Political Doctrines of Antiphon the Sophist : a Reconsideration », Proceedings of the Cambridge Philological Society, N.S. 4, $n^{\circ} 184$ (1956-1957), p. 26-32.

- The Sophistic Movement, Cambridge, 1981. [Trad. fr. par A. Tordesillas \& D. Bigou, Paris, 1999, Bibliothèque des textes philosophiques).]

LABARRIÈre, J.-L. (à paraître) : «L'homme apolitique : pesseia, polis et apolis : Politiques, I, 2, $1253 \mathrm{a} 1-7 \gg$.

LURIA, S. 1927 : «L'argomentazione di Antifonte in Ox. Pap. XV 1797 », Rivista di filosofia, N. S., 5 (1927), p. 80-83.

Mazon, P. 1938 : Homère, Iliade. 3, Chants XIII-XVIII, texte établi et traduit par -, Paris, 1938 (Collection des Universités de France).

MAZZARINO, S. 1966 : Il pensiero storico classico. 1, Bari, 1966.

MORRISON J. S. 1972 : «Antiphon », dans R. K. Sprague (éd.), The Older Sophists : a Complete Translation by Several Hands of the Fragments in Die Fragmente der Vorsokratiker edited by Diels-Kranz with a New Edition of Antiphon and of Euthydemus, Columbia, SC, 1972, p. 106-240.

Moulton, C. 1972 : «Antiphon the Sophist, On Truth», Transactions of the American Philological Association, 103 (1972), p. 329-366.

NArCY, M. 1989 : «Antiphon d'Athènes », dans R. Goulet (dir.), Dictionnaire des philosophes antiques, I, Paris, 1989, p. 225-244.

NeschKe-HentschKe, A. 1995: Platonisme politique et théorie du droit naturel: contributions à une archéologie de la culture politique européenne. 1, Le platonisme politique dans l'Antiquité, Louvain-Paris, 1995 (Bibliothèque philosophique de Louvain, 42).

NiLl, M. 1985: Morality and Self-Interest in Protagoras, Antiphon and Democritus, Leyde, 1985 (Philosophia antiqua, 43).

Ostwald, M. $1990:$ « Nomos and Phusis in Antiphon's Пвpı $\dot{\alpha} \lambda \eta \theta \varepsilon i \alpha \varsigma$ », dans M. Griffith \& D. J. Mastronarde (éd.), Cabinet of the Muses : Essays on Classical and Comparative Literature in Honor of Thomas G. Rosenmeyer, Atlanta, GA, 1990 (Scholars Press Homage Series), p. 293-306.

Pellegrin, P. 1997 : Sextus Empiricus, Esquisses pyrrhoniennes, introduction, traduction et commentaires, Paris, 1997 (Points. Essais, 352).

-2002 : Aristote, Physique, traduction, présentation, notes, bibliographie et index, Paris, $2000,{ }^{2} 2002$ (GF 887).

PENDRICK, G.J. 2002 : Antiphon the Sophist: the Fragments, edited with introduction, translation and commentary, Cambridge, 2002 (Cambridge Classical Texts and Commentaries, 39).

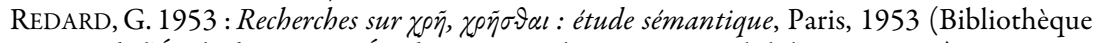
de l'École des Hautes Études. Sciences historiques et philologiques, 303). 
Romeyer-Dherbey, G. 1995 : «Cosmologie et politique chez Antiphon», Bulletin de la Société française de philosophie, 89.4 (1995), p. 105-129.

SAHLINS, M. 2008 : The Western Illusion of Human Nature, with Reflections on the Long History of Hierarchy, Equality and the Sublimation of Anarchy in the West, and Comparative Notes on Other Conceptions of the Human Condition, Chicago, 2008.

STENZEL, J. 1924 : « Antiphon (15) », dans Paulys Realencyclopädie der classischen Altertumswissenschaft. Supplementband 4, Stuttgart-Weimar, 1924.

Stevens, A. 1999 : Aristote, La Physique, introduction de L. Couloubaritsis, traduction de —, Paris, 1999, « Bibliothèque des textes philosophiques ».

THEANDER, C. 1920 : « De fragmentis Antiphontis Sophistae novis », Nordisk tidsskrift for filologi, 4, ser. 9 (1920), p. 1-7.

THOMAS, Y. 2011 : «L'institution juridique de la nature : remarques sur la casuistique du droit naturel à Rome », dans M.-A. Hermitte \& P. Napoli (éd.), Les Opérations du droit, Paris, 2011 (Hautes études), p. 21-40.

UNTERSTEINER, M. 1962 : I sofisti. Testimonianze e frammenti, Fascicolo quarto, Antifonte, Crizia, introduzione, traduzione e commento a cura di A. Battegazzore \& M. Untersteiner, Florence, 1962 (Biblioteca di studi superiori, 7).

VERNANT, J.-P. 1974 : «Le mariage», dans Mythe et société en Grèce ancienne, Paris, 1982 (Fondations), p. 57-81.

WismanN, H. 1980 : «Réalité et matière dans l'atomisme démocritéen 》, Siculorum Gymnasium, 33 (1980), p. 61-74 